\title{
Single-photon avalanche diode imagers in biophotonics: review and outlook
}

\author{
Claudio Bruschini ${ }^{1}$, Harald Homulle${ }^{2}$, Ivan Michel Antolovic ${ }^{1}$, Samuel Burri ${ }^{1}$ and Edoardo Charbon ${ }^{1}$
}

\begin{abstract}
Single-photon avalanche diode (SPAD) arrays are solid-state detectors that offer imaging capabilities at the level of individual photons, with unparalleled photon counting and time-resolved performance. This fascinating technology has progressed at a very fast pace in the past 15 years, since its inception in standard CMOS technology in 2003. A host of architectures have been investigated, ranging from simpler implementations, based solely on off-chip data processing, to progressively "smarter" sensors including on-chip, or even pixel level, time-stamping and processing capabilities. As the technology has matured, a range of biophotonics applications have been explored, including (endoscopic) FLIM, (multibeam multiphoton) FLIM-FRET, SPIM-FCS, super-resolution microscopy, time-resolved Raman spectroscopy, NIROT and PET. We will review some representative sensors and their corresponding applications, including the most relevant challenges faced by chip designers and end-users. Finally, we will provide an outlook on the future of this fascinating technology.
\end{abstract}

\section{Introduction}

Individual single-photon avalanche diodes (SPADs) have long been the detector of choice when deep subnanosecond timing performance is required, due to their excellent single-photon detection and timestamping capability ${ }^{1-4}$. The breakthrough implementation of the first SPADs in standard complementary-metal-oxide semiconductor (CMOS) technology ${ }^{5}$ triggered the exploration and design of large digital SPAD imagers, potentially manufactured in volume at affordable prices. This was soon followed by the first integrated SPAD array ${ }^{6}$ and a host of architectures, ranging from simpler implementations of the early days, based solely on off-chip data processing, to progressively "smarter" sensors including on-chip, or even pixel level, time-stamping and processing capabilities. Modular setups have also been designed, either through the combination of SPAD arrays with FPGAs ("reconfigurable pixels"), or by means of very recent 3D

\footnotetext{
Correspondence: Claudio Bruschini (claudio.bruschini@epfl.ch)

${ }_{1}^{1}$ AQUA, EPFL, Neuchâtel, Switzerland

${ }^{2}$ AQUA, TU Delft, Delft, The Netherlands

These authors contributed equally: Claudio Bruschini, Harald Homulle
}

developments. Furthermore, basically all implementations rely on FPGA-based host boards; combined with the natively digital SPAD data output, this opens the door to real-time algorithmic implementations in close proximity to the sensor, such as FPGA-based autocorrelation and lifetime calculations.

As SPAD technology matured, a range of applications have been explored in very diverse fields, such as consumer and robotics imaging, data and telecom security, advanced driver-assistance systems and biophotonics, which is the main subject of this review. In particular, we will discuss (endoscopic) fluorescence lifetime imaging (FLIM), (multibeam multiphoton) FLIM-FRET (Förster resonance energy transfer), single-plane illumination fluorescence correlation spectroscopy (SPIM-FCS), localisation- and entangled photons-based super-resolution microscopy (SRM), time-resolved Raman spectroscopy, near-infra-red optical tomography (NIROT) and positron emission tomography (PET). However, it is true that SPAD imagers are still mostly used in specialised research settings, apart from some notable non-imaging exceptions, such as SPAD arrays in the form of silicon photomultipliers (SiPMs), which are readily available from a 
number of manufacturers. This is at first glance surprising, given the aforementioned potential for unrivalled photon counting and time-resolved performance; however, it can be partly traced back to some performance parameters that still lag behind those of established CCDs and sCMOS imagers, such as the quantum efficiency over the whole spectrum and the fill factor, which are of importance for several light-starved applications. The pixel sizes are typically larger, limiting so far the manufacturing of megapixel arrays. On the technological side, the design of high performance, low-noise SPADs is challenging; the same is true at the system level for data handling, leading to important firmware development efforts. Therefore, it is not surprising that recent efforts have been focused, at the device level, on increasing the SPADs' key figures of merit ${ }^{7}$ and improving the contact with foundries, to fully profit from possible process optimisations.

In the following sections, which are mostly dedicated to SPAD imagers in standard CMOS technologies, we will first discuss the SPAD state-of-the-art, starting from individual devices, their key properties and the corresponding optimisation trade-offs, which are strongly influenced by the target application. We will then focus on the impact of design choices on the overall sensor architecture and the most important challenges, moving up in a vertical fashion from the pixel level, considering the basic circuitries and in-pixel options, to array architectures (1D vs. 2D) and the readout, which is of particular importance for real-time implementations. A host of biophotonics applications will then be described, starting from FLIM and its different flavours and ending with more disruptive scenarios and sensor concepts such as quantum-based super-resolution microscopy and 3D-stacking (the combination of a top sensor layer with a bottom control and processing layer), respectively.

Interested readers are encouraged to refer to ref. ${ }^{3}$ for details of other applications of SPAD-based imagers and to refs. ${ }^{8-11}$ for a comparison with established devices and alternative CMOS imagers.

\section{SPAD detectors and imagers}

\section{Single-photon avalanche diodes}

Photodetectors capable of measuring single photons have been known for decades and have been realised using different technologies, from photomultiplier tubes (PMTs) to microchannel plates (MCPs) and electronmultiplying charge-coupled devices (EMCCDs). However, the implementation of large, all solid-state single-photon imagers (Fig. 1a) calls for a new kind of miniaturised, scalable device featuring a reliable set of performance parameters. One example of such a device is represented by the SPAD implemented in industry-standard CMOS technology. The SPAD is basically a photodiode whose $\mathrm{p}$ $\mathrm{n}$ junction (Fig. 1b) is reverse biased above its breakdown voltage $V_{\mathrm{bd}}$, such that a single photon incident on the active (i.e. photosensitive) device area can create an electron-hole pair and thus trigger an avalanche of secondary carriers. The avalanche build-up time is typically on the order of picoseconds, so that the associated change in voltage can be used to precisely measure the time of the photon arrival ${ }^{1,12}$. This operation regime is known as Geiger mode; hence, the devices are also known as Geiger-mode APDs (GmAPDs).

The self-sustaining avalanche in the SPAD needs to be stopped as soon as possible to prevent the destruction of the device itself due to the high current. The corresponding quenching occurs by lowering the SPAD bias voltage $V_{\mathrm{OP}}$ below the breakdown voltage, e.g. by using a resistor in series with the SPAD. The voltage across the SPAD then needs to be restored to its initial value above the breakdown voltage, before the next photon can trigger another avalanche. During this interval, which is typically on the order of tens of nanoseconds and known as dead time, the SPAD will be almost insensitive, depending on the exact quenching and recharge mechanism and SPAD front-end implementation. The sensitivity of the SPAD will gradually increase, until it reaches its nominal value when the recharge is complete. The change in voltage across the SPAD during a detection event is easily transformed into a digital signal by using a front-end discriminator, for example a single transistor or an inverter; the discriminator has an important role in determining the minimum detectable avalanche current. The resulting output, which does not depend on the wavelength of the impinging photon, is compatible with standard electronics, which makes it easy to integrate a SPAD into larger circuits and arrays of detectors. Table 1 summarises the most important properties of SPADs and compares them across the SPAD-based imagers reported in Table 2.

A number of parameters are used to describe the performance of a single SPAD device ${ }^{7}$. The most important is the photon detection probability (PDP), which represents the avalanche probability of the device in response to photon absorption at a given wavelength. In CMOS SPADs, the PDP has a peak in the visible region, which can reach $70 \%$ for single, optimised diodes ${ }^{13}$. Other important parameters are the dark count rate (DCR), i.e. the observed avalanche rate in the absence of light, and afterpulsing, which introduces false events that are correlated in time with previous detections ${ }^{7}$. When SPADs are grouped in imagers one must consider electrical and optical crosstalk and the fill factor, which denotes the ratio between the photosensitive area and the total pixel area. Although the fill factor is generally calculated from the drawn area, the actual fill factor might be slightly 

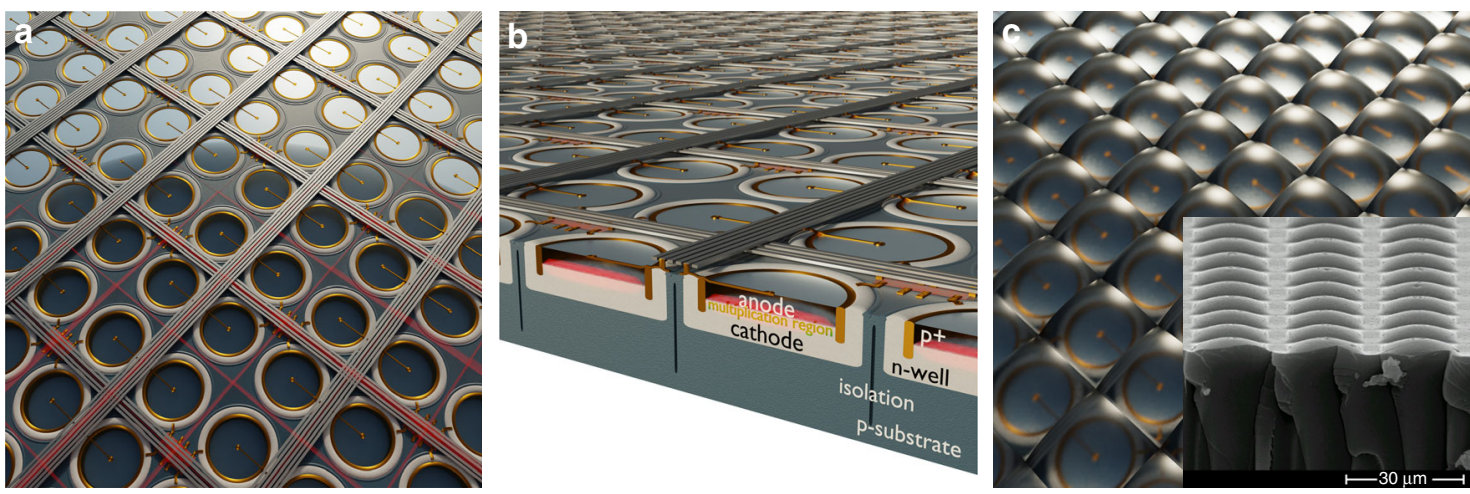

d

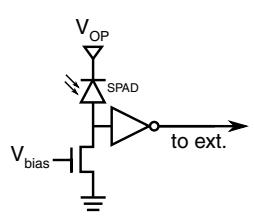

$\mathbf{g}$

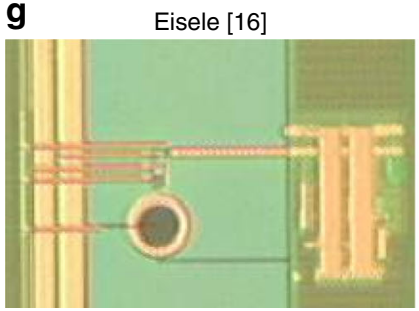

e
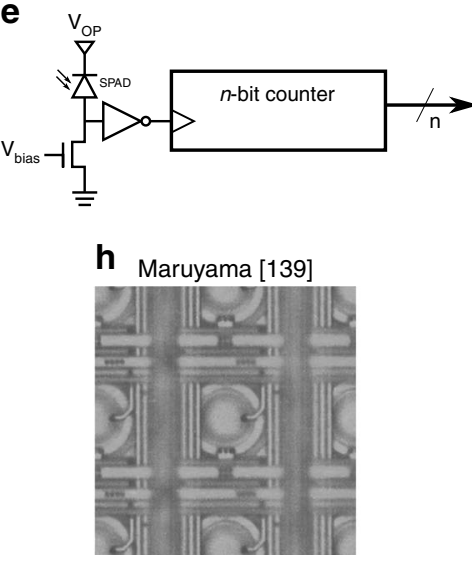

f

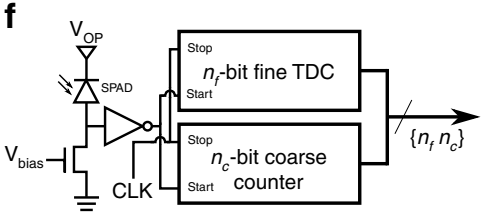

i

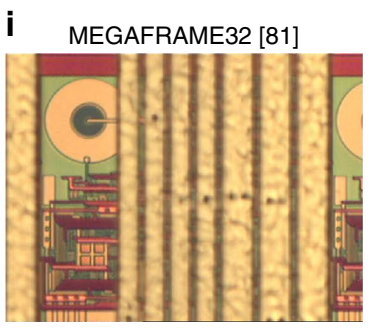

Fig. 1 SPAD arrays and comparison of the SPAD pixel architectures. a Artist's impression of a SPAD array (top view) and $\mathbf{b}$ an example of the corresponding cross-section for a substrate isolated SPAD in a conventional CMOS process, depicting some of the key components (diode anode/ cathode and corresponding p-n junction, multiplication region in which the avalanche is triggered, and the substrate and isolation from it) ${ }^{3}$. The SPAD fill factor can be enhanced with microlenses (c), and the inset shows an SEM image from ref. ${ }^{15}$. The design of individual pixels ranges from $\mathbf{d}$ basic structures, which are only capable of generating digital pulses corresponding to individual photon arrivals on the SPAD, to e pixels including counters, which add the individual arrivals over a given time window that is possibly gated, or $\mathbf{f}$ more advanced electronics such as a complete TDC, which make it possible to time-stamp individual photon arrival times. The corresponding examples of pixel micrographs are displayed in $\mathbf{g}-\mathbf{i}$, as reprinted from refs. $16,81,139$

Table 1 Key SPAD pixel parameters and typical values commonly found in the sensors listed in Table 2

\begin{tabular}{ll}
\hline & Value range \\
\hline SPAD pixel & \\
Dead time [ns] & $10-100$ \\
DCR [cps/ $\left.\mu m^{2}\right]$ & $0.3-100$ \\
PDP (peak) [\%] & $10-50$ \\
Fill factor [\%] & $1-60$ \\
Timing resolution [ps] & $30-100$ \\
Afterpulsing probability [\%] & $0.1-10$ \\
\hline
\end{tabular}

lower, typically by a few percentage points, due to edge effects, leading to an inactive SPAD area (see ref. ${ }^{14}$ for a detailed discussion of the so-called inactive distance). The
SPAD's fill factor obviously affects the overall imager sensitivity, given that it is multiplied by the PDP to give the overall photon detection efficiency (PDE).

Many of the SPAD characteristics can be optimised in the design phase, often requiring trade-offs. For example, a larger size of the guard ring, to better contain the high electric field and prevent a premature edge breakdown, will positively impact the crosstalk, at the expense of the active area and thus the fill factor. This can be compensated with larger diodes at the cost of the DCR, which increases with diode area. A short dead time allows a higher count rate, and thus a high dynamic range, but increases the afterpulsing probability, which leads to problems when detecting photon correlations. The targeted sensor application should ideally be taken into account during the design phase to select the optimal trade-offs, such as sensitivity versus noise and speed versus fill factor. 


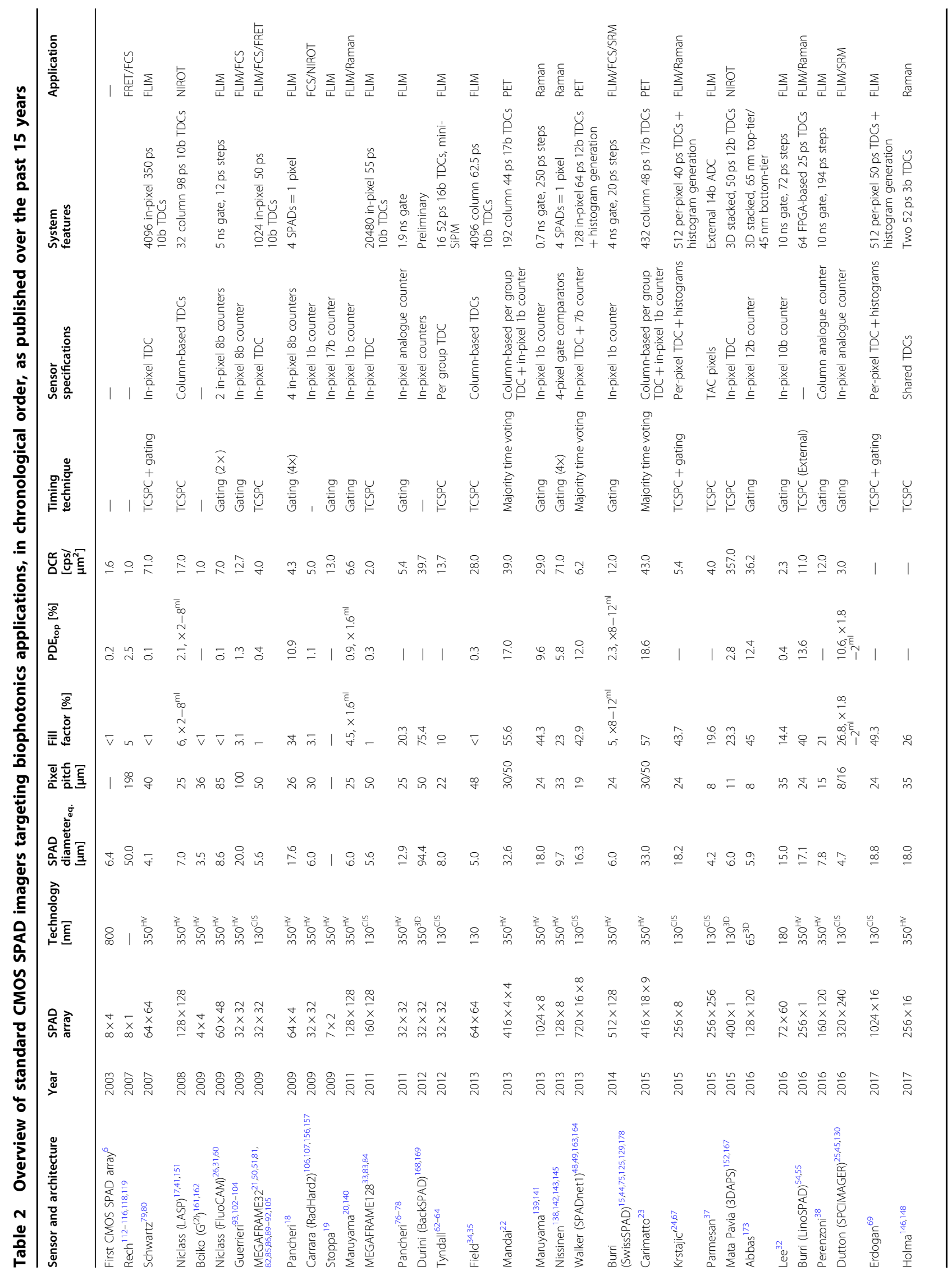




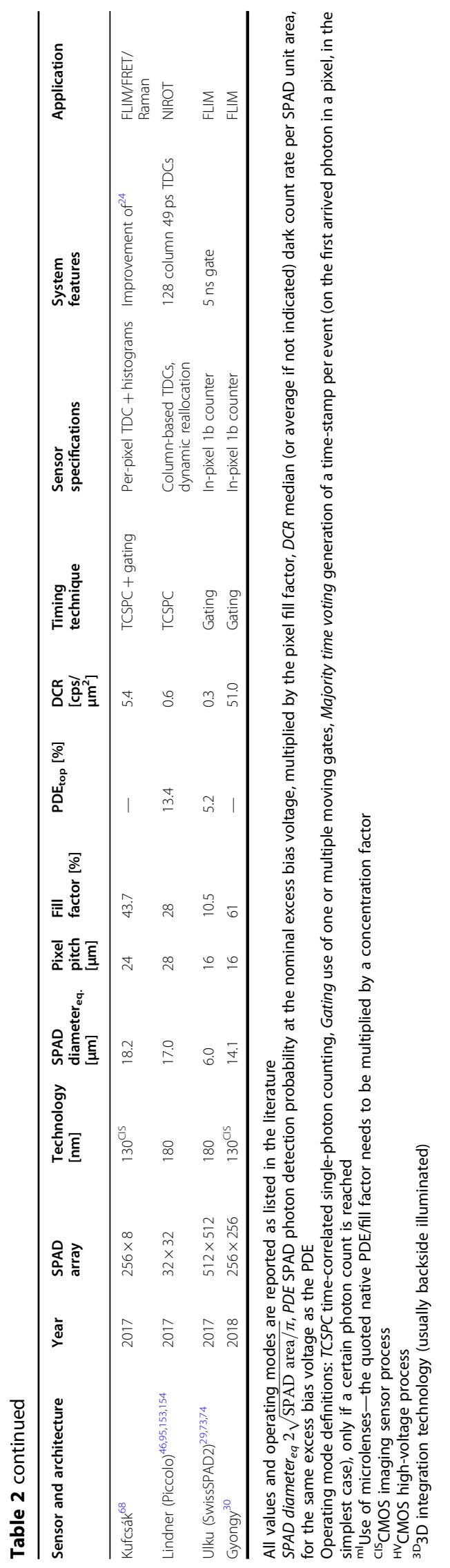

\section{From individual SPADs to arrays}

When a suitable SPAD device and pixel circuit have been demonstrated in a given fabrication process, they can be integrated into an array to form a SPAD imager. The simplest array is linear, allowing the designer to place the detection and processing electronics outside the photosensitive area, thus achieving higher fill factors. A 2D array of pixels, on the other hand, requires selfcontained circuits, in-pixel or at the periphery, to acquire, store and transmit data. This additional circuitry negatively impacts the fill factor, but eliminates the need for scanning to create a complete image. Some freedom also exists at the level of the spatial granularity; grouping pixels, for example, reduces the overall data throughput, while preserving key information, such as photon timing and reducing the complexity. The same is true for the temporal granularity, allowing, for example, the acquisition of only a subset of all possible timestamps for specific applications. Finally, the sensor fabrication itself might include post-processing steps, such as the deposition of microlenses to increase the overall sensitivity (Fig. 1c).

We discuss the various architectural choices and the corresponding trade-offs below, moving from the pixel level up to the array design specificities.

\section{Architectures \\ Pixel architecture}

We divide SPAD pixel circuits into three broad types, depending on the functionality added on top of the basic photon-to-electrical pulse conversion. The first type is represented by a basic structure, which only includes the circuitry necessary for a full detection cycle consisting of the avalanche generation, quench and recharge. The output of such a pixel is a train of electrical pulses corresponding to individual photon detections. The second type is a pixel with built-in counter, consisting of a counting circuit and at least one bit of memory; its output is a photon count. The third type of pixel is timecorrelated and includes circuitry to discriminate the arrival time of photons; its output can be as simple as a flag for a detection during a given time window or as complex as a variable number of timestamps reporting distinct photon arrival times. Concept schematics for the three types of pixels are shown in Fig. 1d-f, while selected implementation examples are displayed in Fig. 1g-i. The pixel fill factor obtained when assembling an array is inversely proportional to the amount of electronics placed besides the SPAD, which makes it advantageous to use modern fabrication technologies that enable smaller feature sizes.

The pixel design elements common to all types of pixels include active quenching and recharging, masking and gating. Active quenching and recharging can be employed to optimise the detection cycle of a SPAD by reducing the 
dead time in a well-controlled manner and thus improving the maximum count rate. In this case, active circuitry can be used to stop the avalanche and recharge it earlier than what is possible with passive resistive approaches. This limits the amount of charges flowing through the diode, improving its lifetime and reducing the afterpulsing. Active techniques for quenching and recharging have been employed in a number of designs ${ }^{12,15-26}$.

It is worth noting that a measured SPAD count actually corresponds to an event with one or more simultaneously detected photons, whose exact number cannot be resolved (SPADs are indeed also termed click detectors ${ }^{27}$ ). Nevertheless, one can still estimate the number of detected photons by using preknowledge about the actual SPAD quenching and recharging mechanism and the temporal distribution, e.g. exponential, of the impinging photons $^{28}$.

Masking is used to selectively disable pixels inside an array. This feature is commonly employed in designs where multiple pixels share circuitry to avoid overloading by particularly noisy pixels, which would otherwise decrease the overall performance. Possible implementations can either switch off the SPAD, thereby preventing avalanches from taking place, or disconnect pixels from the read-out circuit. Switching off the SPAD has the additional benefit of removing possible crosstalk. Examples of pixel architectures with masking are $\mathrm{a}^{20,22-24}$.

Gating is another independent element of pixel architectures and consists of enabling the SPAD only for a limited time, down to picosecond windows. Gating can be applied directly to the SPAD (device gating) or to its front-end electronics (electrical gating). In the case of SPAD gating, either the cathode bias needs to be lowered below the breakdown voltage, or the anode bias needs to be increased above the excess bias; the observation window starts when the SPAD is activated. If the required bias swing is higher than what is applicable in standard CMOS circuitry, external bias control is needed. In this case, however, the gate's rise and fall times are increased due to the capacitance of the external gating line. In contrast, gating the front-end electronics can be directly integrated into the CMOS circuitry, thus enabling sharper gate profiles. The drawback is that the SPAD is still active outside the gate, and has, therefore, to be recharged before opening the gate.

The key advantage of gating is the temporal discrimination that can be achieved through its implementation, even though the overall detection efficiency is reduced. In a setup with repetitive (pulsed) illumination, gating permits the selective capture of photons during a portion of the repetition period, with the added option of shifting the gating window in picosecond steps. This feature can also be used to exclude parts of the photon response that are not of interest. Exemplary gating applications include rapid lifetime determination in FLIM, the accurate reconstruction of a particular optical response in the time domain, the elimination of early/ background-related detections, or the reduction of a sample's intrinsic fluorescence in Raman spectroscopy. Gating can also be used to reduce the DCR by eliminating dark counts occurring outside the time zone of interest.

Moving beyond the basic structures, pixels have been designed to include memory elements for multiple purposes. A single-bit memory can be used to capture a purely binary image during a given time interval (i.e. a frame time), for example when it is important to avoid global shutter artefacts; this kind of architecture can be implemented with only a few transistors, thus still allowing for reasonable native fill factors (5-10\%), while a fast read-out (10-100 kpfs) is usually employed to increase the dynamic range and accumulate multi-bit images off-chip ${ }^{15,20,29}$. Fabricated in a $130 \mathrm{~nm}$ CMOS process, the pixel in ref. ${ }^{30}$ reaches a notable fill factor of $61 \%$ by using an all NMOS design, an analogue storage element, and deep $n$-well sharing between the pairs of SPAD rows, at the expense of a reduction in the timing accuracy due to the simplified gating circuit, and a somewhat increased crosstalk between pixels.

Multi-bit and multiple counters allow differentiation between the number of captured photons. When used together with multiple gates, a simple in-pixel phase detector can be constructed and read-out requirements can be relaxed, while maintaining a good dynamic range. Integrating more memory in each pixel drastically reduces the fill factor and makes it advantageous to move to smaller technology nodes. For example, the $2 \times$ 8-bit counter pixels detailed in ref. ${ }^{31}$, implemented in older $0.35 \mu \mathrm{m}$ technology, result in a fill factor of $0.8 \%$, while the 10-bit counter pixel reported in ref. ${ }^{32}$, designed with a $0.18 \mu \mathrm{m}$ process, results in a fill factor of $14.4 \%$.

Pixels with an integrated arrival time measurement, typically implementing time-to-digital converters (TDCs) or their analogue counterparts (time-to-analogue converters or TACs), represent the most powerful, but also the most complex pixel architecture. The timing circuitry in general needs to be as compact and low power as possible to be integrated in every pixel of an imager, while still offering the required timing resolution. Arrays with in-pixel TDCs usually do not exceed fill factors of a few percent ${ }^{21,33-35}$, with ref. ${ }^{36}$ representing a notable exception, reaching a fill factor of over $19 \%$. A ring-oscillator is typically used for time-stamping with a resolution of tens of picoseconds (fine measurement), whereas the timing range is extended with a counter as needed (coarse measurement). Analogue techniques, such as in-pixel or column-level TACs or analogue memories in the form of 
capacitors, are making a comeback because they can be implemented in area-efficient ways, at the expense of analogue-to-digital converters placed at the periphery of the array or outside of the chip, and the difficulties inherent in mixed-signal design, e.g. non-uniformities and mismatch. Notable examples of sensors using analogue elements are refs. ${ }^{4,25,37,38}$, the last of which presents an array with a fill factor of $26.8 \%$.

\section{Array architecture}

The simplest form of a SPAD pixel array is a single line. In a linear (1D) array (Fig. 2a, d) all pixel electronics is placed outside the sensor area, with only the diode guard ring separating the active area of different pixels. Most linear SPAD arrays allow a truly parallel pixel operation, even if resource sharing is in principle possible in the same way as for $2 \mathrm{D}$ arrays. The $1 \mathrm{D}$ architecture allows to a

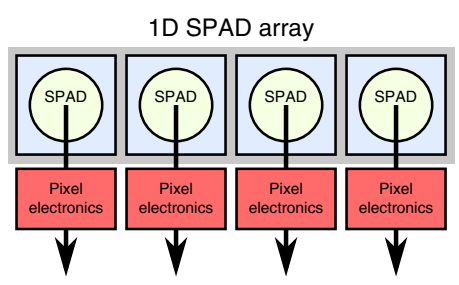

d

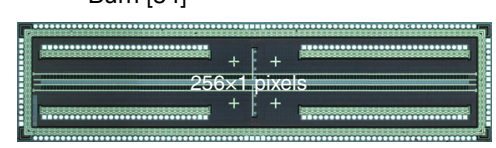

b

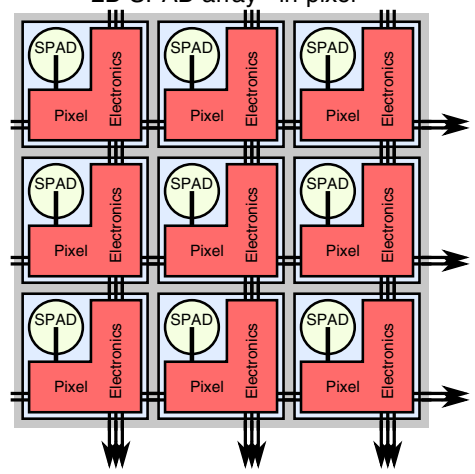

e MEgAFRAME [81]

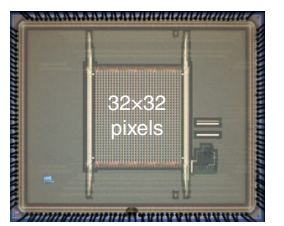

C 2D SPAD array - column-parallel

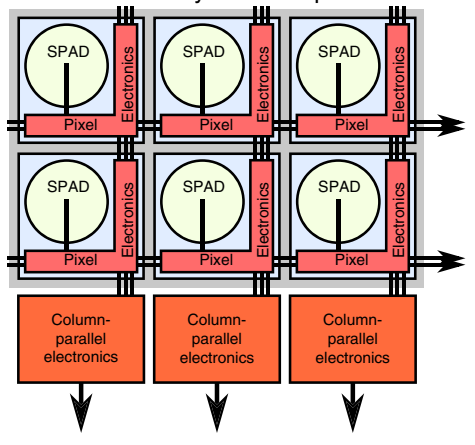

f Field [35]

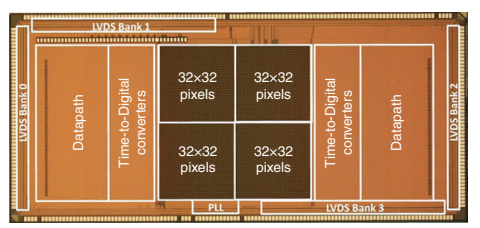

g

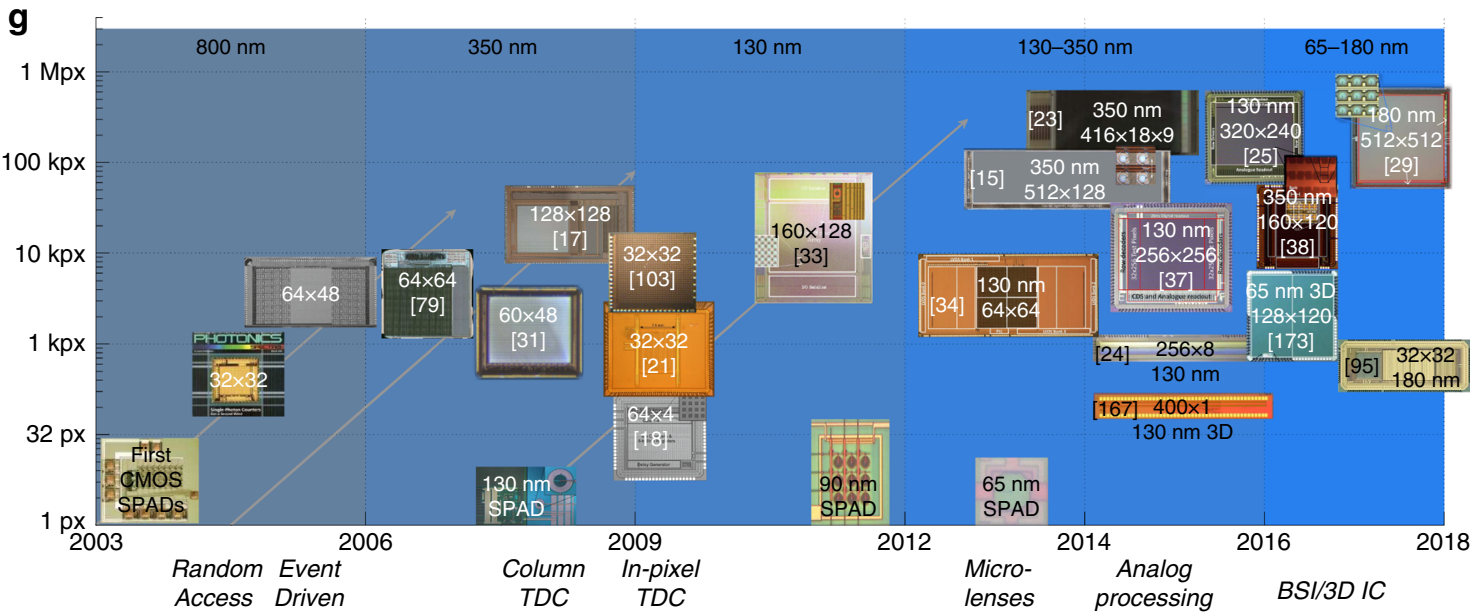

Fig. 2 Comparison of the SPAD array architectures. a In linear arrays, the pixel electronics can be placed outside the pixel area, leading to an increase in the fill factor; in 2D arrays, the fill factors are generally smaller, because $\mathbf{b}$ electronics is needed inside the pixel itself, or at least $\mathbf{c}$ at the array periphery, e.g. for column-based TDCs. The related advantages and disadvantages are discussed in detail in the text, and the corresponding examples of array micrographs can be found in $\mathbf{d}-\mathbf{f}$, as reprinted from refs. ${ }^{35,54,81}$. Finally, $\mathbf{g}$ provides an overview of the evolution of SPAD imagers over the last 15 years in terms of the total number of pixels (on the vertical axis), the technology node (indicated at the top of the image), and some salient architectural characteristics, such as random access or event driven (indicated at the bottom of the image). Only some representative examples, primarily targeted at biophotonics applications, are shown here (details are reported in Table 2). The diagonal lines indicate the developments along a given technology node (800, 350 and $130 \mathrm{~nm}$ ), which are usually started by optimising the SPADs before designing full imagers. Recent years have seen a trend towards higher spatial resolutions and 3D IC solutions 
reach the highest possible fill factors, at the expense of the optical or mechanical scanning solutions that are needed to generate a $2 \mathrm{D}$ image, should this be required by the target application.

Two-dimensional SPAD pixel arrays (Fig. 2b, e) are capable of acquiring 2D images directly, at the expense of a more complex sensor design for the interconnection between pixels and read-out electronics. In general, all supply, control and data signals are shared across the rows and columns of a 2D pixel array to maximise the fill factor. The minimal circuitry needed at the pixel level is a read-out line driver, but usually more circuitry is added, such as gating and counters with memory, as discussed in the previous subsection. More complex pixels include time-stamping electronics and in-pixel photon information counting or timing pre-processing. Depending on the application requirements, some circuit elements, such as complex time-to-digital converters, can be shared among multiple pixels, either for a larger block of pixels or, more commonly, for (multiple) rows or columns (Fig. 2c, f). Non-uniformities and timing skews grow in general with increasing array size, and similarly the overall generated data volume also increases, calling for specific read-out solutions as discussed below. One possibility is to bin the pixels in groups, e.g. in situations where the spatial resolution can be traded off with the signal-to-noise ratio (SNR).

Despite the efforts to maximise the fill factors in 2D arrays, the obtained fill factors are usually below those of similarly sized sCMOS cameras (also see Table 2), especially for complex pixel architectures like in-pixel TDCs, due to the larger transistor counts. Microlenses, therefore, represent a viable option to reclaim some of the fill factor lost due to the electronics. These micro-optical devices are placed in front of the sensitive area, typically on the surface of the detector, and concentrate impinging photons onto the active (i.e. photosensitive) pixel area (Fig. 1c). Examples of SPAD-related microlens developments and sensors are presented in refs. ${ }^{20,39-45}$. The microlenses are typically optimised for specific applications and properties (for example collimation) of the impinging light.

In 2D imagers, it is possible that the pixels no longer strictly operate in parallel, for example when they contain memory elements that are addressed and then reset by the read-out (to gather new photons) on a row-by-row basis (rolling shutter acquisition operation). This can lead to well-known artefacts, such as temporal lag, when recording phenomena with very fast dynamics. Therefore, some imagers implement a true global shutter operation, which provides an image snapshot at a given instant. This can be achieved by activating all pixels together at the start of a frame and then "freezing" the data acquisition at the end of the frame and starting the read-out operation, with some loss in efficiency (reduced temporal aperture). An alternative that does not call for (many) expensive global signals is represented by an event-driven operation mode, which allows continuous on the fly recording of events as they occur; one way of implementing this operation mode is by using a common bus shared by all pixels (e.g. in a column), with separate address lines to identify the SPAD that has fired ${ }^{46}$.

Considering all the trade-offs, encountered when selecting a pixel and array architecture, there is no single best implementation. The architecture of a SPAD array should, therefore, be chosen based on the target application, sometimes even abandoning classical imaging approaches, or at least benefiting from the flexibility provided by SPAD arrays, e.g. by binning pixels and preprocessing data close to the sensor. As an example, we consider time-of-flight positron emission tomography (ToF-PET), where the information of interest is represented by the energy, time-of-arrival and interaction coordinates of gamma rays; the gamma rays are converted by means of scintillating crystals into visible light photons, to be detected by SPAD arrays in the form of SiPMs. In this case, it makes sense to reduce the effective granularity of the recorded data by grouping multiple SPADs together and compensate for noisy detectors using masking. The gamma ray energy is given, in this digital approach, by the total number of SPADs that have fired in a time window of a few hundred nanoseconds, while the time-of-arrival can be estimated on chip and refined by the local control and communication FPGA. An overview of the digital approaches to SPAD-based sensors for PET is provided in ref. ${ }^{47}$, while individual detector architectures are detailed in refs. ${ }^{22,23,48,49}$.

\section{Read-out architecture}

One of the main concerns when interfacing with a single-photon camera is the resulting high data rate, especially when recording timestamps of individual photons or working at very high frame rates. Eventually, the data rate needs to be reduced to a level where it can be transferred to a computer or storage medium. This can be realised, for example, with the same approach as what is used in a streak camera, whereby information captured during a (very) short duration is stored locally (in the pixel) at a high speed, and then read out at a low speed for processing and storage. Another possibility is represented by in situ extraction of higher level information. The corresponding algorithms, such as histogram accumulation or multi-bit count integration, can be implemented on the control FPGA, or even on the sensor itself. In the case of fluorescence lifetime imaging, for example, realtime systems have been devised that can calculate the lifetime of molecules at video rate, without the need for recording the full single-photon data stream $^{9,50,51}$, 
including multi-exponential scenarios ${ }^{52}$. An FPGA system indeed offers some flexibility in terms of possible data processing and a high computational bandwidth, which can be used, for example, to realise firmware-based $32 \times$ 32 autocorrelator arrays as detailed in ref. ${ }^{53}$ (with FCS as the target application). The "reconfigurable pixel" concept proposed in refs. ${ }^{54,55}$ maximises flexibility by moving the whole circuitry, which is usually placed beyond the basic SPAD pixel structure, inside the FPGA; this makes it possible to implement different TDC or counter architectures, with the goal of tailoring the system (sensor and firmware) in an optimal way according to the needs of the target application.

\section{SPAD sensor summary}

Table 2 lists a comprehensive summary of the main SPAD-based sensors and imagers that have been designed for biophotonics applications; they are discussed in the next section, together with the related applications and the corresponding results. A representative subset is shown in Fig. 2g, which provides a graphical overview of how these imagers have evolved over the last 15 years.

\section{Biophotonics applications}

The following sections analyse in detail a host of biophotonics applications that have been explored with SPAD imagers, starting from FLIM, which has been addressed early on, and its different flavours, ending with more disruptive scenarios and forward looking sensor concepts. The use of SPADs in these applications is summarised in Table 3 and compared to the use of nonSPAD-based methods, highlighting the SPAD benefits and drawbacks, ongoing technology developments and selected experiments.

\section{Fluorescence lifetime imaging}

Fluorescence lifetime imaging (FLIM) is a non-invasive measurement technique for applications where the fluorescence intensity does not provide sufficient information or discrimination. Common usage of FLIM is found in the study of living tissues and cells at the molecular level, because the fluorescence lifetime is insensitive to fluorescence intensity and to the corresponding probe concentration, at least to a reasonable extent ${ }^{56}$; however, the samples should not be subject to excessive illumination intensities, to avoid phototoxicity and photobleaching. Other advantages are the detection of lifetimes that can be dependent on $\mathrm{pH}$, temperature, oxygen concentration and viscosity levels, thereby enabling the detection of effects that cannot be observed with simple fluorescence intensity measurements.

The slow acquisition speed $(<10 \mathrm{~Hz})$ is the main limitation of standard FLIM setups. While the photophysics at the molecular level contributes to this, the detection system can also impose major speed limitations. Timecorrelated single-photon counting (TCSPC), which requires timestamps of individual photons, is often the detection method of choice due to its very high precision, but the underlying hardware and data acquisition are hard to scale to large multichannel arrays; scanning is, therefore, required when TSCPC is used in an imaging setup. Time-gated sensors have also been employed, including large sensitive areas; they rely on one or more (moving) gates to recover the timing information, and thus the lifetime, at the expense of a reduction in the overall sensitivity, as discussed in the "Architectures" section.

Interested readers can refer to refs. ${ }^{9,10,57-59}$ for the background literature on FLIM and the related sensors, techniques and applications. In the following subsections, we will focus on standard CMOS SPAD implementations for FLIM and how these implementations have been engineered and employed to address the aforementioned limitations.

\section{Point-like FLIM}

Point-like FLIM systems offer increased signal-to-noise ratios by combining the individual outputs of several pixels. An example of such an approach is represented by the FluoCam ${ }^{26}$ system, which comprises a $60 \times 48$ SPAD array $^{31}$, with two 8-bit time-gated counters in each pixel. The gates can be externally programmed to shift in steps of $\sim 12 \mathrm{ps}$, to cover a full laser repetition period with high accuracy. The two counters, therefore, allow a precise reconstruction of the fluorescence response, even when significant photobleaching distorts the signal, including sub-nanosecond lifetimes. The integration times are on the order of several minutes, but can be substantially reduced by resorting to more recent designs and/or technology nodes.

The FluoCam system has been used in several in vivo studies to demonstrate the capabilities of such an approach, employing indocyanine green (ICG)-modified derivatives, such as ICG-RGD, which target the $\alpha_{\mathrm{v}} \beta_{3}$ integrin; the final goal was to explore the feasibility of surgical applications with exogenous NIR targeted fluorophores $^{26,60}$. The system was capable of discriminating between healthy (muscle and tail) and cancerous tissues in a mouse with a glioblastoma mouse model, even though the lifetime difference was only $\sim 50 \mathrm{ps}(10 \%$ level in this case) between the lifetimes of the bound and unbound fluorophores as shown in Fig. 3a.

In TCSPC mode, the detection system's limitations introduce pile-up effects for detection rates $>0.1$ photon/ laser cycle, causing an underestimation of the lifetime. Pile-up correction using standard SPAD detectors has been discussed in detail by Léonard et al. ${ }^{61}$, who show that low count rates are not necessarily needed to avoid pileup, at the expense of an increase in the lifetime estimation 


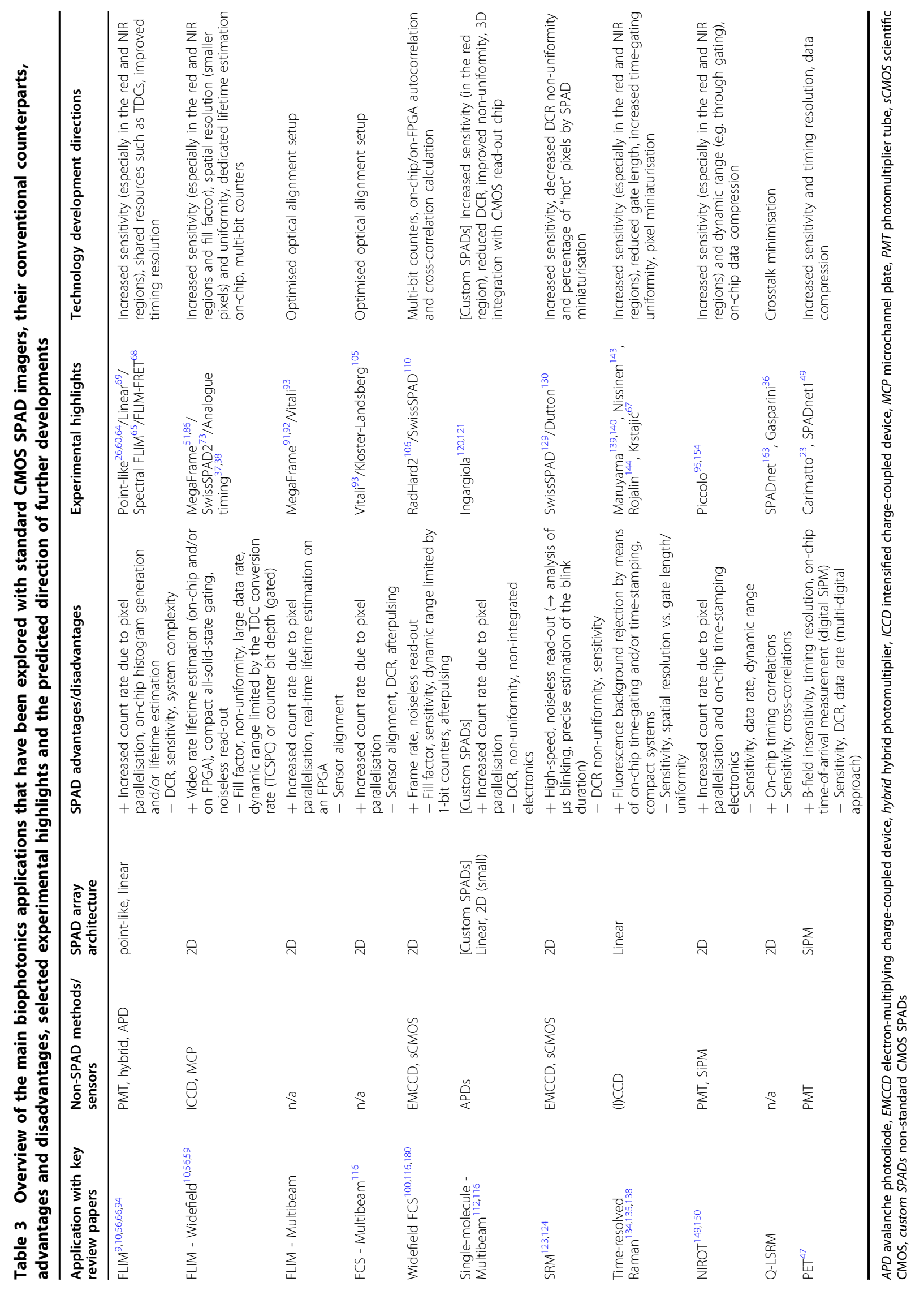



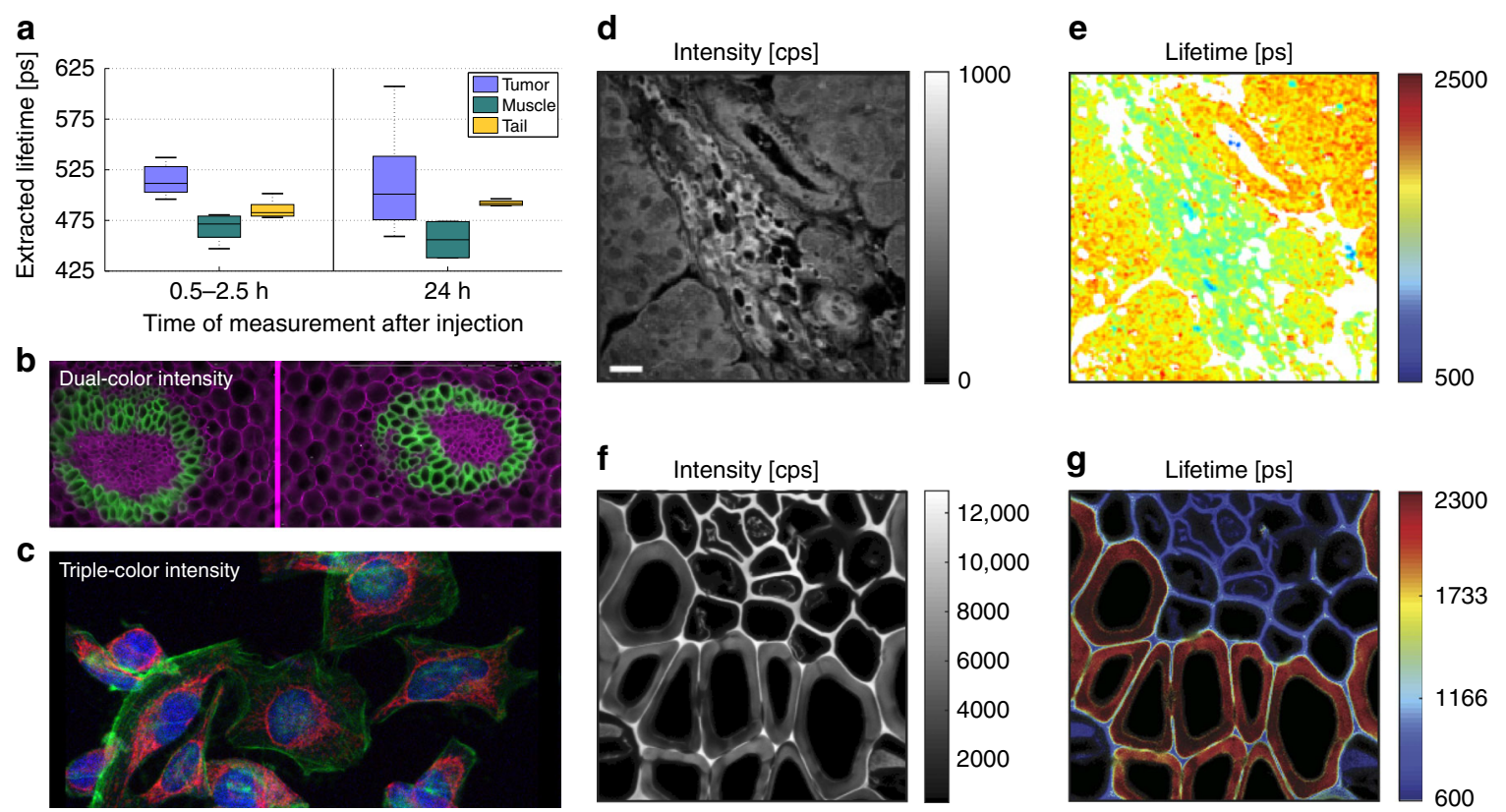

Fig. 3 Example fluorescence intensity and/or lifetime results. a FluoCam system used in a point-like mode for the study of monomeric ICG-C (RGDfK) injected in a mouse with a glioblastoma mouse model. A subtle lifetime shift between tumour and non-tumour tissue is observed ${ }^{26}$. $\mathbf{b}$ Dualcolour intensity fluorescence image of a thin slice of a plant root stained with a mixture of Safranin and Fast Green, taken with the SwissSPAD widefield time-domain gated array ${ }^{178}$. c Triple-colour intensity fluorescence image of HeLa cells labelled with DAPI, Alexa 488 and Alexa 555, taken with SwissSPAD2 ${ }^{73}$. d, e Label-free FLIM of an unstained liver tissue excised from a tumourigenic murine mode ${ }^{65}$, imaged with a $64 \times 4$ SPAD array ${ }^{18}$. f, g A Convallaria FLIM measurement performed with a linear $32 \times 1$ SPAD array ${ }^{70}$. The images are reprinted from refs. ${ }^{26,65,70,73,178}$

variation. In integrated SPAD detectors, multiple subsystems contribute to pile-up. A detailed analysis of the influence of the SPAD, timing and routing dead time on the lifetime estimation can be found in ref. ${ }^{62}$, with experimental results based on a mini-silicon photomultiplier $(32 \times 32$ SPAD pixels $)$ implemented in a $130 \mathrm{~nm}$ CMOS process, and featuring an on-chip lifetime estimator $^{63,64}$. The outputs of all pixels are routed towards 16 TDCs that can time-stamp up to 8 photons per excitation period. These timestamps are then processed in a centre-of-mass module to derive the fluorescence lifetime. This technique has been validated with reference samples with relatively long lifetimes of over $1 \mathrm{~ns}$, demonstrating that reliable lifetimes can be estimated, with a proper architecture, at photon count rates that go well beyond the classical pile-up limit.

Linear SPAD arrays and corresponding FLIM applications

The advantages and disadvantages of linear architectures have already been discussed at the beginning of the Array architecture section. Pancheri and Stoppa ${ }^{18}$ implemented a $64 \times 4$ linear SPAD array (overall size of $1660 \times 104 \mu \mathrm{m}^{2}$ in $0.35 \mu \mathrm{m}$ CMOS technology) targeted for FLIM. The four SPADs in each column were connected to the same read-out channel, creating macropixels to reduce the influence of the single SPAD dead time ( $\sim 50 \mathrm{~ns})$. This increased the photon throughput of a $15.8 \times 63.2 \mu \mathrm{m}^{2}$ macro-pixel. The chip also featured four time-domain gates that were connected to four separated counters, enabling the construction of on-chip histograms of the photon-arrival times with four bins and data compression. This sensor was later used ${ }^{65}$ for spectrally resolved FLIM (sFLIM or $\lambda$ FLIM), a setup that enables the separation of molecules by both the fluorescence emission wavelength and the fluorescence lifetime ${ }^{66}$. An example of a corresponding tissue image is shown in Fig. 3d, e. The $\lambda$ FLIM system simplifies the discrimination of different fluorophores and enables the simultaneous study of donor and acceptor molecules.

More recently, Krstajić et al. presented a linear $256 \times 2$ SPAD array with a pixel pitch of $23.7 \mu \mathrm{m}$ and a high fill factor of $43.7 \%$, implemented in a $130 \mathrm{~nm}$ CMOS process $^{24,67,68}$. Each pixel was connected to one TDC with a 40 ps LSB. The sensor also featured an on-chip centre-ofmass $(\mathrm{CMM})$ calculation for mono-exponential fluorescence lifetimes, enabling to output lifetimes at a $200 \mathrm{~Hz}$ line rate with up to $65 \mathrm{kphotons} /$ pixel (limited by the SPAD dead time). Alternatively, the chip can output perpixel TCSPC histograms with a 320 ps bin resolution. Multicolour microspheres and skin autofluorescence lifetimes were measured, with a data acquisition time of $5 \mathrm{~min}$ for TCSPC data, to be compared with 2 or $200 \mathrm{~ms}$ 
when operating in CMM mode for fluorophores in cuvette or skin autofluorescence, respectively. This chip is currently being used in the (https://www.proteus.ac.uk/) EPSRC UK PROTEUS project, targeting in vivo, in situ microendoscopic instrumentation for the diagnosis of lung diseases. Erdogan et al. ${ }^{69}$ designed a next generation linear array, in the same $130 \mathrm{~nm}$ CMOS process, extending the resolution to $1024 \times 8$. The chip featured 512 TDCs and on-chip histogram generation that decreases the output data rate and mitigates the $\mathrm{I} / \mathrm{O}$ and USB bottlenecks.

Linear arrays can also be used as single-point detectors, for example by means of optical 1D to 2D transformations, to reduce the effect of single SPAD dead time and increase the throughput in FLIM measurements ${ }^{70}$; an example of the corresponding results is shown in Fig. 3f, g. This and other approaches could pave the way for highthroughput biotechnological applications, such as highthroughput screening or cell sorting ${ }^{61,71}$, based on nanosecond-lived fluorophores.

\section{Widefield time-domain gated FLIM}

Gated SPAD arrays are in principle easier to implement over large areas than TCSPC solutions, and thus provide an appealing path to all-solid-state widefield, timeresolved imaging.

An initial implementation of a time-gated $128 \times 128$ SPAD array with 1-bit memory combined an on-chip 600 ps delay line and an off-chip 200 ps delay line for gate shifting $^{20}$. DNA molecules labelled with Cy 5 were placed directly on the chip surface and the lifetime was measured. Time-gating enabled an excitation elimination without the need for dichroic mirrors.

SwissSPAD ${ }^{15}$ is a $512 \times 128$ SPAD array with an in-pixel 1-bit memory and time-gating capability. The 1-bit frames are read out at $156 \mathrm{kfps}$, while time-gating enables independent global exposures as short as $4 \mathrm{~ns}$. The gate position can be shifted in 20 ps steps with respect to a reference signal. This enables the reconstruction of the exponential lifetimes per pixel. The implemented pixel, which contains 12 transistors, has a 5\% fill factor due to the $0.35 \mu \mathrm{m}$ manufacturing process. With the use of microlenses, the effective fill factor is increased to $50-60 \%$ for collimated light, as often featured in microscope output ports ${ }^{44}$; a representative fluorescence intensity image is shown in Fig. 3b.

Early characterisation of SwissSPAD (without microlenses) for FLIM measurements was performed with reference data sets ${ }^{72}$; the sensor could properly extract the lifetime of fluorophores in the nanosecond range. Ulku then designed SwissSPAD's successor, SwissSPAD2 29,73,74, a $512 \times 512$ SPAD array-the largest time-resolved SPAD image sensor to date-with a higher PDP and a lower DCR, based on a similar architecture. A triple-colour fluorescence intensity image is shown in Fig. 3c. Further research on widefield time-domain gated FLIM with microlenseenabled versions of SwissSPAD architectures is ongoing, including real-time phasor-based measurements $\mathrm{s}^{73,75}$.

Following up on the proof-of-concept work by Pancheri et al. ${ }^{76-78}$, Perenzoni et al. ${ }^{38}$ designed a $160 \times 120$ SPAD imager with gating, but with multi-bit memory. The gate can be set as short as $750 \mathrm{ps}$, with rise and fall times down to $200 \mathrm{ps}$, and a frequency of $50 \mathrm{MHz}$. Instead of a 1-bit memory, this chip uses an analogue counter, enabling multiple photon accumulations per frame at the cost of introducing ADCs. The pixel pitch is $15 \mu \mathrm{m}$, resulting in a $21 \%$ fill factor in $0.35 \mu \mathrm{m}$ high-voltage CMOS technology. Gyongy et al. ${ }^{30}$ pursued an $n$-well shared pixel approach to achieve a high native fill factor of $61 \%$ for a $256 \times 256$ SPAD array with $4 \mathrm{~ns}$ gates, 600 ps fall times with an onchip delay generator, and a pixel pitch of $16 \mu \mathrm{m}$.

\section{Widefield TCSPC FLIM}

A $64 \times 6440 \mu \mathrm{m}$ pitch pixel array designed in $0.35 \mu \mathrm{m}$ high-voltage CMOS technology, featuring 64 columnparallel TDCs and a timing resolution of $\sim 350 \mathrm{ps,}$, represented an early implementation of a SPAD TCSPC array work $^{79,80}$. However, the maximum PDE of $0.1 \%$ was relatively low.

The MEGAFRAME32 high-performance sensor was smaller $(32 \times 32$ SPADs) but adopted a radically different architecture, based on $50 \mathrm{ps,} \mathrm{10-bit} \mathrm{in-pixel} \mathrm{TDCs,}$ working at a maximum rate of $500 \mathrm{kfps}^{21,81}$, and recording either time-correlated data (one time-stamp per pixel), or time-uncorrelated data (6-bit counting). In the former operation mode, up to 0.5 billion timestamps could be generated per second ${ }^{82}$. The fill factor $(1 \%)$ was adversely affected by the large in-pixel electronics; on the positive side, this demonstration stimulated pioneering microlens research to bring the fill factor back up. MEGAFRAME32 was extensively employed to explore bio-applications and subsequently extended to a $160 \times 128$ array (MEGAFRAME128), adding peripheral intelligence (data compression and CMM pre-processing $)^{33,83,84}$.

Gersbach et al. reported early high frame rate FLIM proof-of-concept investigations ${ }^{21,85}$ with MEGAFRAME32. Li et al. ${ }^{50,51,86}$ illustrated how firmware-based rapid lifetime estimation algorithms, such as CMM (centre-of-mass method), make full use of the large number of available timestamps to enable video-rate (50 fps) real-time FLIM operation. An example of the corresponding in vivo two photon FLIM data, with both the intensity and the lifetime, is shown in ref. ${ }^{86}$ using an FITC-albumin probe, which was injected into a rat bearing a P22 tumour and measured 100 min after the injection. A clear distinction between the blood vessels and the tumour tissue could be observed in the lifetime image (biexponential decay), in contrast to the intensity image. 
Another widefield FLIM application of the same sensor, coupled to DNA microarrays, was reported in ref. ${ }^{87}$ employing a TIRF excitation geometry. Distinct lifetime signatures, corresponding to dye-labelled $\mathrm{HCV}$ and quantum-dot-labelled HCMV nucleic acid targets, could be distinguished over 320 pixels, with concentrations as low as $10 \mathrm{nM}$ and an exposure time of $26 \mathrm{~s}$.

A different architecture was selected by Field et al. for their $64 \times 64$ array, in a standard $0.13 \mu \mathrm{m}$ CMOS process, and reported in ref. ${ }^{34,35}$, namely with one TDC per pixel (LSB of $62.5 \mathrm{ps)} \mathrm{placed} \mathrm{at} \mathrm{the} \mathrm{column} \mathrm{level;} \mathrm{this} \mathrm{led} \mathrm{to} \mathrm{a}$ pixel pitch of $48 \mu \mathrm{m}$. The sensor was aimed at video-rate operation (100 fps), but the corresponding extreme data rate of $42 \mathrm{Gbps}$ led to a high power consumption (14.5 mW/pixel).

Parmesan et al. ${ }^{37}$ have chosen to emphasize small pixel pitches $(8 \mu \mathrm{m})$, while still maintaining a pixel fill factor of nearly $20 \%$, by resorting to an architecture based on inpixel time-to-amplitude (TAC) converters, with a global ramp voltage. This enabled the design of a large $256 \times 256$ array, which could work either interfaced to external TDCs (with optimal timing performance, but resulting in a slower system) or using an on-chip coarse flash ADC (with a lower temporal resolution).

\section{Multibeam FLIM}

Multibeam architectures enable increased photon throughput and reduced FLIM acquisition times. Coelho et al. and Poland et al. used MEGAFRAME32 with a spatial light modulator (SLM) for multibeam multiphoton FLIM, increasing the throughput by the number of parallel beamlets ${ }^{88-91}$. The fill factor does not decrease the sensitivity in such setups, because beams are concentrated onto the active area of the SPADs. FLIM data of live cells (MCF-7 human carcinoma cells) labelled with green fluorescent protein were acquired within $500 \mathrm{~ms}$, albeit at a reduced accuracy. This approach was extended in ref. ${ }^{92}$ with a new CMM method, mostly implemented in hardware, which was capable of pixel level background subtraction and did not require prior knowledge of the expected lifetime; real-time operation was obtained, with a reduced accuracy compared to the accuracy of TCSPC mean squared fitting techniques.

Vitali et al..$^{93}$ also used a multibeam approach with a $32 \times 32$ array (square pixels of $100 \mu \mathrm{m}$ with a $20 \mu \mathrm{m}$ circular SPAD and 8-bit counters) and performed FLIM of eGFP in living HEK293-T cells. The SPAD sensor was implemented in a standard CMOS process and included time-gating with a minimum gate width of $1.5 \mathrm{~ns}$ and delay steps below $100 \mathrm{ps}$.

\section{FLIM-FRET}

FRET uses interactions between two different chromophores (light-sensitive molecules) that non-radiatively transfer energy from a donor to an acceptor molecule. The energy is transferred only when the distance between the two molecules is small enough ( $\mathrm{nm}$ scale, establishing long-range dipole-dipole coupling) and when there is sufficient overlap between the emission spectrum of the donor and the excitation spectrum of the acceptor. During this coupling, one can observe a decrease in the donor fluorescence and an increase in the acceptor fluorescence. A typical application is the study of protein-protein interactions and the measurement of distances between molecular groups in protein conformations ${ }^{94}$.

FLIM-FRET not only measures the fluorescence intensity change in the donor and acceptor emissions, but also the shortened lifetime of the donor molecule as a result of quenching ${ }^{56}$. By measuring the ratio between the quenched and non-quenched lifetime, the donor-acceptor interactions can be quantified independently from the molecule concentrations within a diverse sample (in contrast to emission intensity FRET).

Poland et al. implemented a MEGAFRAME32-based multifocal FLIM-FRET detector system combined with the optical setup mentioned in the Multibeam FLIM section $^{88-91}$. While scanning protein-protein interactions in live cells with a frame time of $500 \mathrm{~ms}$, they studied changes in FRET interactions between epidermal growth factor receptors (EGFR) and adapter proteins Grb2, as well as a ligand-dependent association of HER2-HER3 receptor tyrosine kinases. Kufcsák et al. ${ }^{68}$ used 5carboxyfluorescein as a donor and methyl red as an acceptor in FRET for thrombin detection. Thrombin cleaves the connection between the donor and acceptor, separating them in space and removing the energy transfer.

\section{Conclusions}

The applicability of SPAD arrays for FLIM was limited in the early implementations by the relatively low PDP and fill factor (multibeam FLIM being an exception to the latter, due to the corresponding peculiar optical setup), combined with high a DCR. While increasing the sensitivity in the red and NIR regions is still important on the SPAD development roadmap, substantial progress has been made in all performance metrics, as also summarised in the overall "Conclusions" section and in Table 3.

SPAD arrays in standard CMOS technology have a clear advantage when parallelising data acquisition is important (including in widefield setups) and/or when real-time operation, e.g. a lifetime calculation, is needed. In general, it is more difficult to operate SPAD arrays than (scanning) single-point detectors, which in addition can be optimised for a maximum sensitivity and/or temporal resolution.

SPAD arrays with resource parallelisation can be used as single-point detectors to reduce the effect of dead time and increase the throughput in FLIM measurements. On- 
chip histogram generation and lifetime estimation further reduce the data rate. This could pave the way to highthroughput biotechnological applications, such as highthroughput screening or cell sorting ${ }^{61,71}$, based on nanosecond-lived fluorophores.

$1 \mathrm{D}$ and $2 \mathrm{D}$ arrays can also eliminate scanning in one and two dimensions, respectively. In this case, an increase in the throughput compared to that of scanning systems is obtained if the non-scanning system dead time T1 is shorter than the array resolution $\mathrm{R}$ multiplied by the scanning system dead time $\mathrm{T} 2$, i.e. $\mathrm{T} 1<\mathrm{R} \times \mathrm{T} 2$. This approach enabled, for example, video-rate imaging lifetime estimation ${ }^{51}$.

Compact, all-solid-state time-domain gated implementations have emerged at the forefront of high-spatial resolution widefield FLIM. Ongoing work is expected to further improve the gate timing precision ${ }^{73}$, whereas an additional increase in the imaging speed will likely occur by implementing multi-bit per-pixel counters. Recent $2 \mathrm{D}$ arrays targeting widefield TCSPC FLIM have increasingly shared TDC resources to combine a higher photon throughput with reduced area and power consumption ${ }^{46,95,96}$.

On the application side, the precision of the lifetime estimation depends on the number of detected photons and the photon efficiency of the used instrumentation and method, which can be characterised by the $F$ value ( $F=\sqrt{N} \frac{\sigma_{\tau}}{\tau}$, where $N$ is the number of detected photons, $\tau$ is the lifetime and $\sigma_{\tau}$ the lifetime estimation precision) ${ }^{97}$. "Ideal" TCSPC systems are widely assumed to have $F=1$, whereas TCSPC SPAD arrays have reported $F$ values up to 1.5 (refs. ${ }^{51,98}$ ), and the most recent time-domain gated implementations have estimated $F$ values between 2 and 5 (refs. ${ }^{74,98,99}$ ). If, for example, a $5 \%$ lifetime estimation precision is required (e.g. 100 ps for a 2 ns lifetime), such as for demanding applications like FLIM-FRET, one can estimate the required number of detected photons with $N=\frac{F^{2}}{0.0025}$. Therefore, a system with an $F$ value of 2 would need each pixel to acquire $4 \times$ more photons than an ideal system to achieve an equivalent lifetime estimation precision, e.g. 1600 photons instead of 400 to reach a $5 \%$ level.

Therefore, higher $F$ values increase the constraints for demanding live-cell imaging, which imposes limitations on both the excitation intensity (due to photobleaching) and excitation duration (due to movement). Reaching a reasonable frame rate, e.g. $10 \mathrm{fps}$, with a gated widefield array calls for a maximum count rate capability of $\sim 160$ kcps per pixel $\left(\frac{1600 \text { detected photons }}{100 \mathrm{~ms}} \times 10\right.$ to avoid read-out pile-up when working with binary frames) to meet the previously mentioned $5 \%$ precision level. Such a requirement is in the range of what is achievable with recent time-domain gated arrays ${ }^{15,30,73,74}$. A similar conclusion can be reached for TCSPC SPAD arrays, such as refs. ${ }^{46,51,95}$, whereas larger implementations call for onchip intelligence, such as histogram generation ${ }^{96,98}$, to relax the constraints on the read-out bandwidth. Note that these estimations assume that the application setup delivers a sufficient photon flux to the sensor, and that the latter features a PDE high enough to reach the requested number of "detected photons" per image frame.

\section{Fluorescence correlation spectroscopy}

Fluorescence correlation spectroscopy (FCS) measures fluorescence intensity fluctuations in time, with the aim of estimating the concentration and diffusion coefficients of fluorophores, including in live cells. These parameters are extracted from the autocorrelation of the temporal intensity fluctuations. Faster sensors and imagers enable the study of smaller and faster molecules ${ }^{100}$. In a widefield setup, the correlation between signals from distant volumes measures the direction and velocity of the flow between the volumes under investigation.

\section{Multibeam FCS}

The multibeam parallelisation principle introduced in the Multibeam FLIM section can also be applied to FCS setups, generating a large number of laser foci using SLMs or diffractive optical elements (DOEs), while taking care to minimise the background signal generated by out-offocus light. A single SPAD or a group of SPADs are then used to detect fluorescence from each laser focus. Goesch et al. used a small, fully integrated $2 \times 2$ CMOS SPAD array in the pioneering work on multibeam FCS reported in ref. ${ }^{101}$. The multibeam concept was later extended to $8 \times 8$ spots to image bright $100 \mathrm{~nm}$ diameter fluorescent beads in solutions using a $32 \times 32$ SPAD array ${ }^{102}$. The latter was then employed, together with the $32 \times 32$ multibeam setup previously described in ref. ${ }^{93}$ to perform FCS of quantum-dot diffusion in solution ${ }^{93,103,104}$. The researchers used a DOE to generate $32 \times 32$ spots with a pitch of $100 \mu \mathrm{m}$ and a diameter of $12.5 \mu \mathrm{m}$ in the image plane (to match the sensor dimensions).

Independently, Kloster-Landsberg et al. ${ }^{105}$ used the $32 \times 32$ MEGAFRAME32 sensor to perform multifocal FCS with live cells, employing a frame time of $2 \mu$ s. In this setup, $3 \times 3$ laser foci were used for experiments with free eGFP in HeLa cells. A larger multibeam array could not be employed due to the high crosstalk between closely packed spots that emerge in this kind of setup.

\section{Widefield SPIM-FCS}

FCS coupled with single plane illumination microscopy (SPIM-FCS) enables a faster characterisation of 3D samples, and records intensity fluctuations over a widefield plane. By illuminating a micrometre-thick light sheet in the $z$-section, the out-of-focus light, photobleaching and photodamage can be minimised. The first SPIM-FCS data 


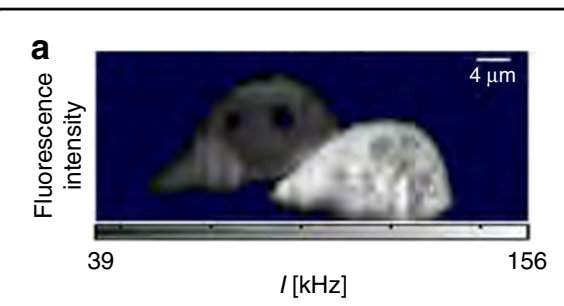

d

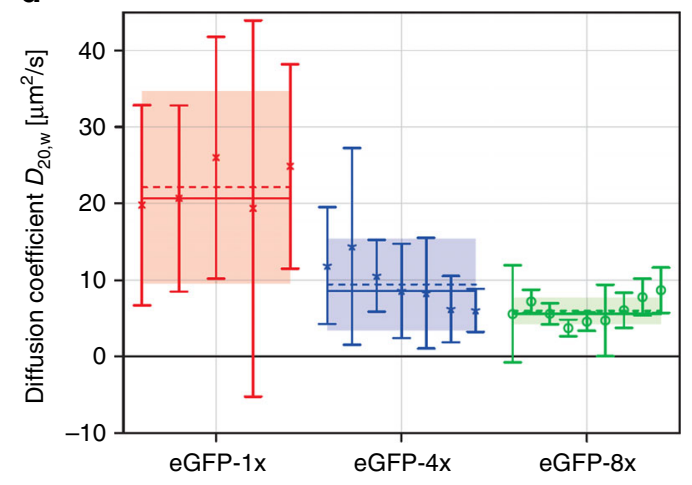

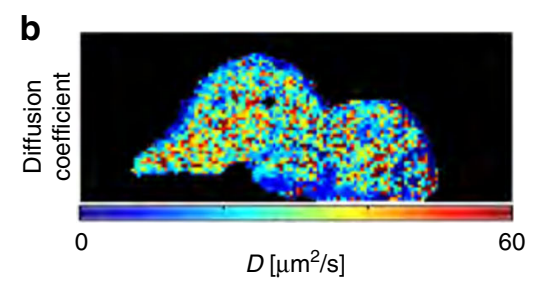
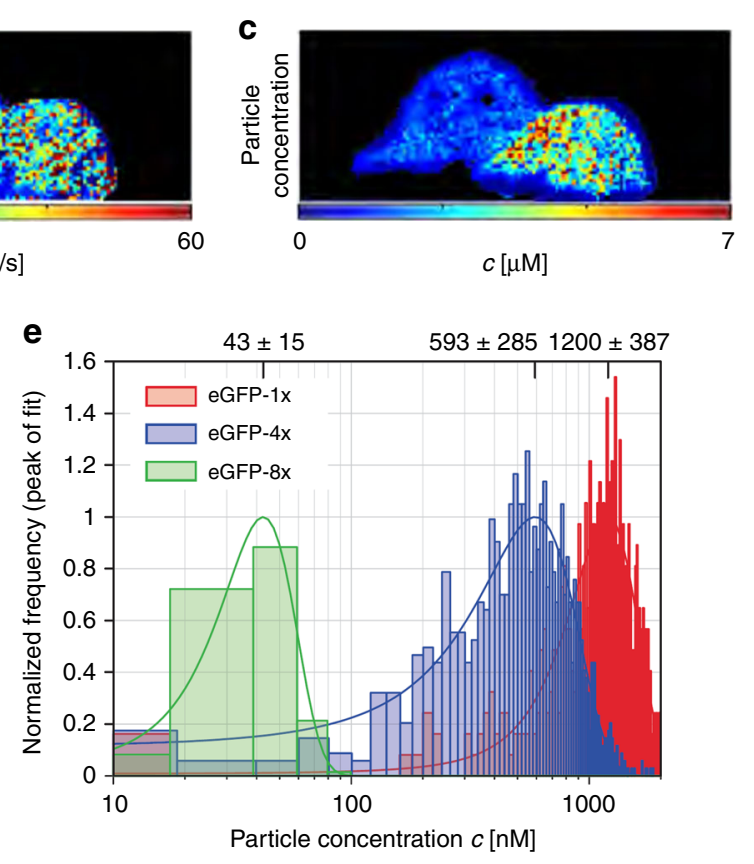

Fig. 4 Widefield SPIM-FCS images of monomeric eGFP oligomers in HeLa cells as recorded with a SwissSPAD widefield imager. a Fluorescence intensity, $\mathbf{b}$ diffusion coefficient and $\mathbf{c}$ dye concentration. $\mathbf{d}$ Diffusion coefficients for three HeLa cells expressing different oligomers. e Particle concentration for the three HeLa cells with different oligomers. The images are reprinted from ref. ${ }^{110}$

obtained with a SPAD array were presented in ref. ${ }^{106}$ The work compared the RadHard2 $32 \times 32$ SPAD array ${ }^{107}$ with EMCCD and sCMOS cameras. The RadHard2 camera achieved very high read-out speeds, up to $300 \mathrm{kfps}$, enabling the extraction of diffusion coefficients down to $3 \mu$ s with a better precision than the EMCCD and sCMOS cameras. The camera was coupled with a realtime $32 \times 32$ autocorrelator, implemented on an FPGA ${ }^{53}$, which allowed real-time autocorrelation calculation for small molecules in solution, with diffusion coefficients down to $10 \mu \mathrm{s}$. However, RadHard2 did not feature microlenses, which obviously affected the overall sensitivity and limited its in vivo applicability.

Widefield in vivo SPIM-FCS with SPAD arrays was first demonstrated with a microlensed version of SwissSPAD $^{15,44,72}$ by Buchholz et al. and Krieger et al. ${ }^{108-110}$. The FCS results in HeLa cells are shown in Fig. 4 for three different oligomers of eGFP. The autocorrelation curves of these measurements featured afterpulsing-like increased correlations at short time lags; these artefacts were mitigated by using spatial cross-correlations ${ }^{111}$, which also allowed the determination of absolute diffusion coefficients without a prior calibration. Although the sensitivity needs to be further increased, this work showed that SPAD arrays can measure protein diffusion in live cells with a better SNR than EMCCD cameras and a minimum lag time of $10^{-5} \mathrm{~s}$. The correlation algorithms were also extended to GPUs.

\section{Conclusions}

The early works demonstrated how multibeam FCS can overcome the limitation of a low native fill factor by concentrating laser foci on the active area of the SPADs. The main challenge in such systems is the optical alignment. Widefield SPIM-FCS has benefited from the high frame rate and noiseless read-out of large binary SPAD arrays, leading to a minimum lag time of $10 \mu \mathrm{s}$. Although microlenses were demonstrated to be effective, a further increase in the PDP is needed to improve the detector's sensitivity, particularly for in vivo measurements. Additional CMOS-enabled functionalities, such as on-chip/onFPGA autocorrelation functions, might assist in reducing the data rate. Finally, low afterpulsing is also a key requirement for FCS; if necessary, the spatial resolution can be reduced by employing cross-correlation of $2 \times 1$ or $2 \times 2$ pixels to eliminate the effect of afterpulsing.

\section{Single-molecule techniques}

Single-molecule fluorescence spectroscopy exploits a low concentration regime to excite individual molecules in a very small volume, and collect rare, burst-like fluorescence emission events corresponding to the transit of individual molecules (whereas in FCS the concentration is such that $\sim 1$ or more molecules are present in the excitation volume at any time-see ref. ${ }^{112}$ for a thorough, SPAD-oriented analysis). The number of generated photons is small (a few dozen or less in a large fraction of 
bursts) and, therefore, the detection is quite challenging. This in turn imposes stringent requirements on the photosensor(s), in terms of sensitivity (including in the red region, where standard CMOS is not advantageous due to the low absorption of silicon), fast response time, low noise (read-out or DCR for SPADs) and high count rates, in order to separate successive or nearby molecules. The measurement times are usually long to accumulate sufficient statistics.

Several (small) arrays of devices fabricated in custom technologies have been employed over the years with success ${ }^{2,112-117}$, and parallelisation strategies have again been adopted to increase the overall throughput, often employing sophisticated optical setups (multispot excitation and detection). For example, a parallel 8-spot singlemolecule FRET (smFRET) analysis system was described in refs. ${ }^{118,119}$ using 8-pixel SPAD arrays and in ref. ${ }^{120}$, where the authors tackled real-time kinetic analysis of the promoter escape by bacterial RNA polymerase, confirming results obtained by a more indirect route.

An extension to 48 excitation spots and two 48-pixel SPAD arrays was detailed in ref. ${ }^{121}$, employing two excitation lasers to separate species with one or two active fluorophores. Apart from successfully tackling the multispot setup issues, the resulting smFRET capabilities were shown for a set of doubly labelled double-stranded DNA oligonucleotides with different distances between the donor and acceptor dyes along the DNA duplex. The resulting acquisition times were drastically reduced to seconds. This could in turn enable high-throughput screening applications and real-time kinetics studies of enzymatic reactions, potentially propelling singlemolecule analysis from a niche technology to a mainstream diagnostic and screening tool ${ }^{120}$.

For a comprehensive overview of this application, where (small) arrays of SPAD devices fabricated in custom technologies dominate, we refer the interested readers to Table 3.

\section{Localisation-based super-resolution microscopy}

The optical resolution is fundamentally limited by diffraction, whereby Abbe defined the corresponding limit to be $\frac{\lambda}{2 n \sin \theta}$, where $\lambda$ is the emission wavelength, $n$ is the refractive index and $\theta$ is the half-angle of the cone of light that enters into the objective ${ }^{122}$. Several super-resolution techniques have emerged over the years to overcome the diffraction limit, enabling resolution enhancements from initial values of $200 \mathrm{~nm}$ down to $10 \mathrm{~nm}^{123}$. One such technique uses sparsely activated (blinking) fluorescent molecules for single-molecule localisation ${ }^{124}$, whereby the location of each molecule is determined by the centre of its point-spread function. The final pointillistic image is then reconstructed by merging thousands of frames with hundreds of thousands of localisations, with a resolution limited by the localisation precision and labelling density. Owing to the long frame sequences, durations of tens of seconds to minutes are necessary to reconstruct the final super-resolved image; this time could be shortened by employing higher frame rates and/or stronger laser intensities.

Antolovic et al. ${ }^{125}$ demonstrated the first localisationbased super-resolution (SRM) images obtained with SPAD arrays, which are compared in Fig. 5a to EMCCD (Fig. 5b) and widefield (Fig. 5c), by employing the SwissSPAD imager ${ }^{15}$. Microlenses were deposited on the SPAD array to improve the fill factor from the native 5 to $60 \%{ }^{44}$, which was a key enabler for applications in which the sensitivity is critical. The image resolution was analysed with the Fourier ring correlation method ${ }^{126}$, yielding a resolution of $\sim 100 \mathrm{~nm}$. The estimated localisation uncertainty $^{127}$ was $30 \mathrm{~nm}$, with 200 photons per localisation, compared to $15 \mathrm{~nm}$ obtained with an EMCCD camera, using 1800 photons per localisation. In other terms, although almost $10 \times$ more photons were acquired with the EMCCD camera, the localisation results were only $2 \times$ better; the reason was that the SNR, which should indeed increase by $\sqrt{10}$, was reduced by $\sqrt{2}$ due to the multiplication noise in the EMCCD camera itself, which arises from the electron multiplication process. Later results with an improved buffer ${ }^{128}$, shown in Fig. $5 \mathrm{~g}$, h, yielded 800 photons per localisation with a localisation uncertainty of $10 \mathrm{~nm}$ for an sCMOS camera, while SwissSPAD collected 100 photons under the same conditions, leading to an uncertainty of $20 \mathrm{~nm}^{129}$.

When compared to standard EMCCD and sCMOS cameras, SPAD imagers eliminate the read-out noise by utilising a direct photon-to-digital conversion-the digital nature of SPAD imagers enables fast and noiseless readout. These properties of SPAD imagers were used in three ways. First, Gyongy et al. ${ }^{130}$ used temporal oversampling and "smart" aggregation to determine the start and end of fluorophore blinking in a more accurate manner to minimise background noise. Second, Antolovic et al. ${ }^{129}$ investigated the performance of the localisation algorithm for different frame rates. Finally, SPAD imagers were used to perform a widefield analysis of fluorophore blinking in the $\mu$ s range ${ }^{129}$.

In terms of the first point, Gyongy et al. used the $320 \times$ 240 SPAD SPCImager ${ }^{25}$ for localisation-based superresolution imaging ${ }^{45,130}$. By employing microlenses, they improved the effective fill factor from 27 to $50 \%$ and used this sensitivity enhancement to demonstrate a resolution of $40 \mathrm{~nm}$. A GATTA-PAINT $40 \mathrm{G}$ nanoruler was used as a reference, and the corresponding results are shown in Fig. 5d-f. "Smart" aggregation decreased the localisation uncertainty by $20 \%$, and the simulations showed a potential improvement by $50 \%$. Although the SPAD results have a localisation uncertainty comparable to that 

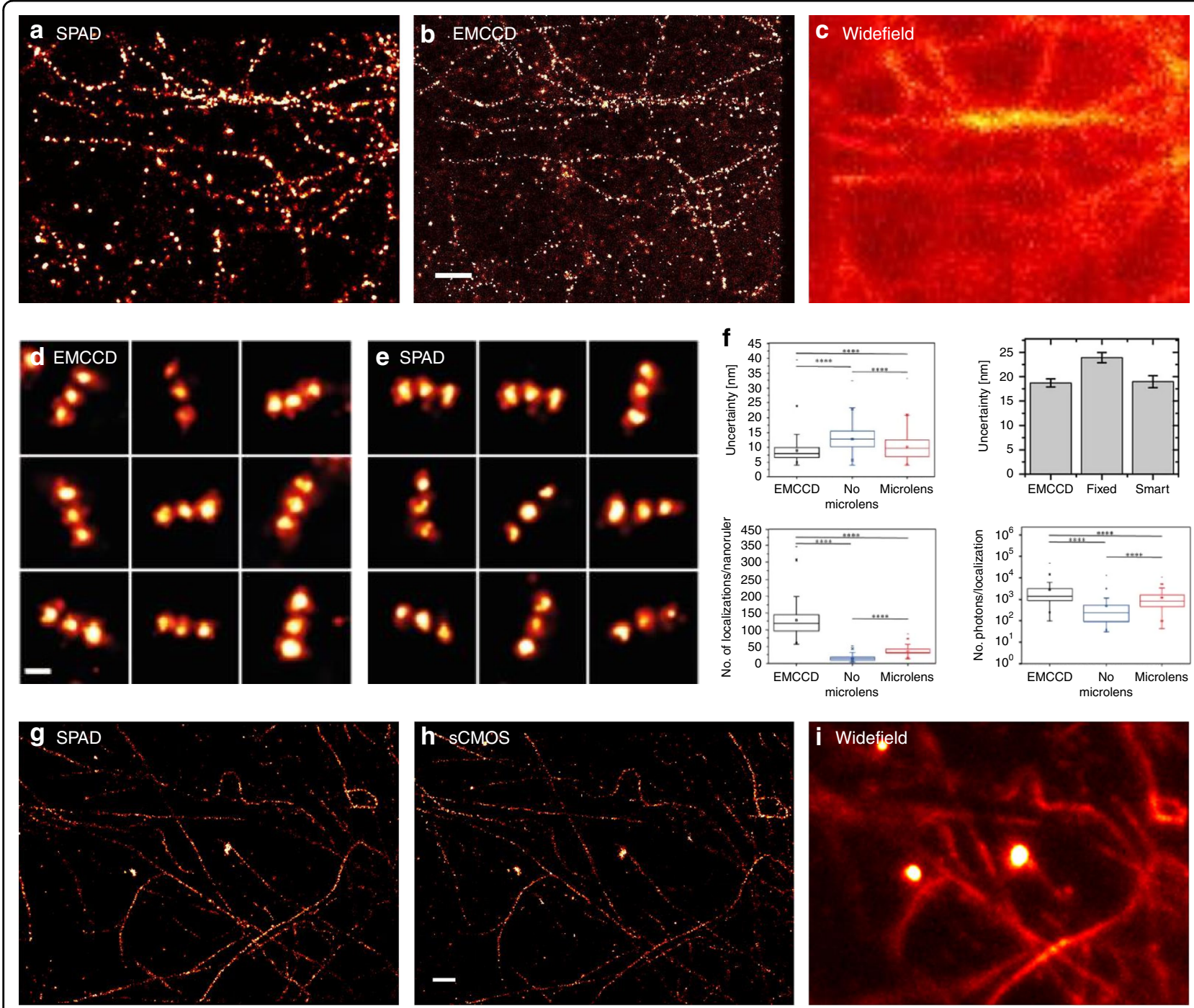

Fig. 5 SPAD super-resolution images. a The first super-resolution image captured with SwisSSPAD, compared to $\mathbf{b}$ EMCCD and $\mathbf{c}$ widefield images. The images show the microtubuli of an U2OS cell labelled with Alexa Fluor 647, in Vectashield ${ }^{129}$. d, e Comparison of the SPCImager using "smart" aggregation and microlenses with an EMCCD. The images show multiple GATTA-PAINT 40G nanoruler localisations ${ }^{45}$. $f$ Comparison of the differences in localisation uncertainty with and without "smart" aggregation and the impact of the microlenses ${ }^{45,130}$. g SwissSPAD super-resolution image of microtubuli labelled with Alexa 647 in OxEA buffer compared to $\mathbf{h}$ sCMOS and $\mathbf{i}$ widefield images ${ }^{129}$. The white bar indicates $1 \mu \mathrm{m}$. The images are reprinted from refs. ${ }^{45,129,130}$

of EMCCDs, a $3 \times$ lower sensitivity in the green leads to a lower number of localisations.

Concerning the second point, Antolovic quantified the optimum frame rate given the exponential distribution of fluorophore blinking ${ }^{129}$, if oversampling and smart aggregation could not be used. Oversampling leads to a higher localisation uncertainty, while undersampling causes a decrease in the number of localisations.

Finally, SwissSPAD's high frame rate of $156 \mathrm{kfps}$ was used to explore additional high-frequency $\mu$ s blinking, which in general reduces the total number of collected photons and thus the final resolution. As an example, the percentage of Alexa 647 molecules blinking at a high frequency decreased from $>68$ to $<30 \%$ when switching from an MEA to an OxEA buffer ${ }^{129}$. This indicated that high-speed SPAD imagers could be beneficial for fluorophore/buffer design and optimisation.

\section{Conclusions}

As the previous discussion has indicated, the fundamental differences between EMCCD, sCMOS and SPAD imagers do also need to take into account the different noise contributions and achievable frame rates, in addition to the overall sensitivity. A theoretical comparison 
between these imagers, when applied to localisation-based super-resolution microscopy, has been conducted by Krishnaswami et al. ${ }^{131}$. EMCCDs feature a quantum efficiency of $90 \%$, but also exhibit multiplication noise that effectively reduces this value by half ${ }^{132}$; the noise performance of EMCCDs is very uniform due to the serial readout, but the achievable frame rate is low. The sCMOS cameras parallelise the read-out and increase the frame rate at the expense of mismatches in the analogue electronics. SPAD imagers eliminate read-out noise with fully parallel digitisation and can thus achieve very high frame rates for binary (1-bit) frames. If multiple 1-bit frames are added to achieve an 8- to 12-bit depth, which might be necessary or not depending on the application, their frame rates become comparable to those of the sCMOS cameras. On-chip counters will eliminate this bottleneck, enabling high frame rates with a higher bit depth. In addition, the high speed and picosecond temporal resolution of TDCs connected to SPADs can open up new avenues, such as video-rate localisation super-resolution and multicolour imaging. The SPAD digitisation also limits the noise contributions solely to the DCR (and afterpulsing), and although DCR levels are acceptable for super-resolution applications, the percentage of "hot" pixels needs to be further reduced.

\section{Raman spectroscopy}

Raman spectroscopy provides data on the chemical composition and molecular structure of a compound in a non-destructive and label-free manner ${ }^{133-135}$, with applications to both in vitro and in vivo tissue diagnostics. This "fingerprinting" technique relies on inelastic light scattering with vibrating molecules, whereby Stokes-scattered Raman photons are redshifted. Raman spectroscopy has seen a surge in biophotonics applications in the last couple of decades ${ }^{134}$, although it typically suffers from a weak scattering cross-section, leading to long overall acquisition times (unless used, for example, in combination with other sampling techniques that reduce the area to be interrogated, line scanning, or multifocal/widefield setups). As such, the Raman signal is often overshadowed by the sample (auto)fluorescence itself; when working with biological samples, it might, therefore, be of interest to move towards the NIR range, where autofluorescence is weaker, although the resulting SNR needs to be weighted by the corresponding reduction in the scattered intensity, which decreases with the 4th power of the excitation wavelength. The use of coherent techniques such as nonlinear anti-Stokes Raman scattering (CARS), resulting in blueshifted radiation, can enhance the scattered signal and circumvent these limitations, enabling, for example, rapid chemical imaging, at the expense of appropriate tunable, femtosecond laser sources ${ }^{134}$. Penetration into deep tissues, which is of particular relevance for in vivo medical diagnosis or when looking at live cells growing in $3 \mathrm{D}$ cultures, can be enhanced with recently developed methods such as spatially offset Raman spectroscopy (SORS) and transmission Raman spectroscopy (TRS); interested readers can consult a recent review ${ }^{136}$.

A disadvantage of moving to the NIR region to reduce the (auto)fluorescence background is that the sensitivity of standard CCD/CMOS imagers decreases, and this is particularly true for SPAD imagers developed with standard CMOS technology, i.e. the primary focus of this review. However, SPADs allow the design of compact, all solid-state detectors for Raman spectroscopy operating in time-resolved mode, whether via very short gates, ideally in the $10-100$ ps range given the nature of the Raman signal (very fast emission compared to the fluorescence background, which is typically in the ns range $)^{137,138}$, or based on time-stamping, e.g. with TDCs; this mode of operation also reduces the DCR contribution and thus enhances the overall SNR. Several linear SPAD-based systems have indeed been recently developed, usually comprising one or a few lines, targeting initially applications such as mineralogy and subsequently biophotonics.

One of the largest reported SPAD arrays is a $1024 \times$ 8 system $^{139-141}$, which was applied with success to mineral samples, where the background fluorescence timescale is longer than for typical biological samples of interest to us. The array featured 1-bit counters and a global gate as short as $750 \mathrm{ps}$ (with a corresponding standard deviation of $120 \mathrm{ps}$ ), which could be shifted in 250 ps steps. The use of a standard $0.35 \mu \mathrm{m}$ CMOS process led to a maximum PDE of $9.3 \%$ with a median DCR of $5.7 \mathrm{kcps}$ at an excess bias of $3 \mathrm{~V}$.

A different approach was chosen in refs. ${ }^{137,142,143}$, where a $2 \times(4) \times 128$ SPAD array, again in a standard $0.35 \mu \mathrm{m}$ CMOS process, featured 4 sub-ns time gates during which the on-chip electronics counted the number of detected photons. This architecture also allowed the determination of the level of the residual fluorescence and DCR in addition to the Raman signal. The fill factor of each SPAD was 23\%, the minimum time gate width was $80 \mathrm{ps}$, and its variation along the spectral axis (timing skew) was \pm 17.5 ps for a nominal width of $100 \mathrm{ps}$; the effect of inhomogeneities of this order in the samples of interest for biophotonics applications (featuring ns- and sub-ns scale fluorescence backgrounds), although small, was also discussed. The overall instrument response function (IRF) was reported to be $250 \mathrm{ps,} \mathrm{and} \mathrm{Raman}$ spectra of several drugs of interest were acquired with a ps pulsed laser at $532 \mathrm{~nm}^{144}$, enabling the authors to reveal previously unseen Raman spectral features. The same underlying standard CMOS technology was used to design a gated $4 \times 128$ SPAD array featuring an additional 512-channel 3-bit flash $\mathrm{TDC}^{145}$, which allowed high timing resolution measurements $(78 \mathrm{ps}, 10 \mathrm{ps}$ standard 
deviation in the first four bins) at the beginning of its $3.5 \mathrm{~ns}$ measurement range. Several SPADs were again employed at a given spectral point, to reduce the impact of noisy pixels.

Further inhomogeneity studies have been carried out by the same group, including a study employing a $256 \times 16$ SPAD test array with two on-chip TDCs, leading to a detailed investigation of the effect of the DCR and PDE on one hand, and gate length variations and temporal skews on the other. The latter can indeed have an important role, with growing design complexity, on the derivation of the Raman spectrum when targeting 100 ps accuracy levels ${ }^{146,147}$. In general, the spectra have been found to be subject to a larger deterioration, as could be expected, when the fluorescence lifetimes and levels become shorter and higher, respectively. An efficient post-processing method relies on a calibration with a known smooth (with respect to the wavelength) fluorescence background; this approach was discussed in ref. ${ }^{148}$, using a highprecision TDC $(<50 \mathrm{ps})$ whose range exceeded the expected timing skew, and sampling a part of the fluorescence spectrum.

The linear $256 \times 2$ array by Krstajić et al. $^{24}$, which was already mentioned in the FLIM section, has also been used for surface-enhanced Raman spectroscopy (SERS), again within the (https://www.proteus.ac.uk/) PROTEUS project. Significant hardware and software improvements of the same sensor were reported in ref. ${ }^{68}$, whereas the removal of fluorescent background signals (in addition to DCR) was illustrated in ref. ${ }^{67}$.

\section{Conclusions}

SPAD arrays have a unique position in time-resolved Raman spectroscopy, offering, in a compact, all solidstate system, a high spatial resolution, integrated gating and/or photon time-stamping in the sub-nanosecond range for fluorescence background rejection. From this perspective, it is surprising that time-resolved SPAD arrays have not yet seen a more widespread use for this application. This is partly due to the sensitivity of the SPADs, which degrades rapidly towards the NIR region, limiting their applicability in further reducing the autofluorescence background by moving to longer excitation wavelengths. Developments are thus heavily focused on the improvement of the sensitivity in the red and NIR regions, and further reducing the gate length and increasing the gate uniformity for biological specimens.

\section{Optical tomography}

Near-infra-red optical tomography (NIROT) studies the absorption and scattering of light in turbid media, e.g. biological tissue. Light that has propagated in a medium is detected at its surface. The measurement process is performed at multiple wavelengths to exploit the knowledge of the absorption and scattering properties of the constituent absorbers. This prior knowledge can then be used to make a $3 \mathrm{D}$ reconstruction of the distribution of these absorbers within the medium; this enables, for example, a non-invasive determination of oxygenation values, usually by working in the $650-850 \mathrm{~nm}$ window (enhanced tissue penetration).

The corresponding image reconstruction inverse problem is, however, ill-posed by nature, leading to a low spatial resolution $(1 \mathrm{~cm}$ level) with existing PMT-based NIROT systems; the latter have seen little use in clinical settings, and are difficult to scale up. The spatial resolution can be improved by increasing the measured amount of information, i.e. ideally, the amount of detectors and their temporal information in the case of time-domain systems. This has led to interest in solid-state detectors and in particular SPADs ${ }^{149}$, either as single devices or in the form of SiPMs, potentially enabling contactless setups and optimised reconstruction algorithms.

While SiPMs can indeed be combined to form a widearea detector ${ }^{150}$, separate TCSPC electronics are still required, and cost might also be an issue. Another route has, therefore, explored fully integrated CMOS SPAD arrays featuring a high PDE and timing accuracy, with thousands of detection channels. An overview of the topic is provided, for example, in refs. ${ }^{41,151,152}$, which also include application examples based on the LASP $128 \times$ 128 TCSPC array ${ }^{17}$. The latter employed 3210 -bit 98 ps column-based TDCs in a pulsed (time-domain) setup recording the time-of-flight information.

An enhanced CMOS SPAD sensor optimised for NIROT was described in refs. ${ }^{95,153}$, targeted at addressing the slow acquisition time bottleneck of previous implementations, which can lead to motion artefacts and decreased patient comfort. This $32 \times 32$ SPAD array, called Piccolo, employed 128 TDCs, based on the concept of dynamic TDC reallocation to reduce the timing-related die area, while simultaneously reducing the probability of photon pile-up. It is implemented in a $180 \mathrm{~nm}$ process employing a large spectral range SPAD and coupled to a cascoded passive quenching circuit to boost the pixel's PDE, reaching a PDP greater than $10 \%$ at $800 \mathrm{~nm}$ with a native fill factor of $28 \%$ at a pixel pitch of $28.5 \mu \mathrm{m}$. The initial results indicated a minimum image acquisition time of $3.8 \mathrm{~s}$ per source and wavelength pair. The sensor has been integrated into a system combining a supercontinuum laser and an acousto-optical filter for a multiwavelength excitation, and a fibre switch to obtain up to 24 source positions ${ }^{154}$ (Fig. 6a); phantom measurements are in good agreement with simulations (Fig. 6b). Future implementations could employ a SPAD gating feature to reduce the impact of the early backscattered photons in certain illumination geometries ${ }^{155}$. 

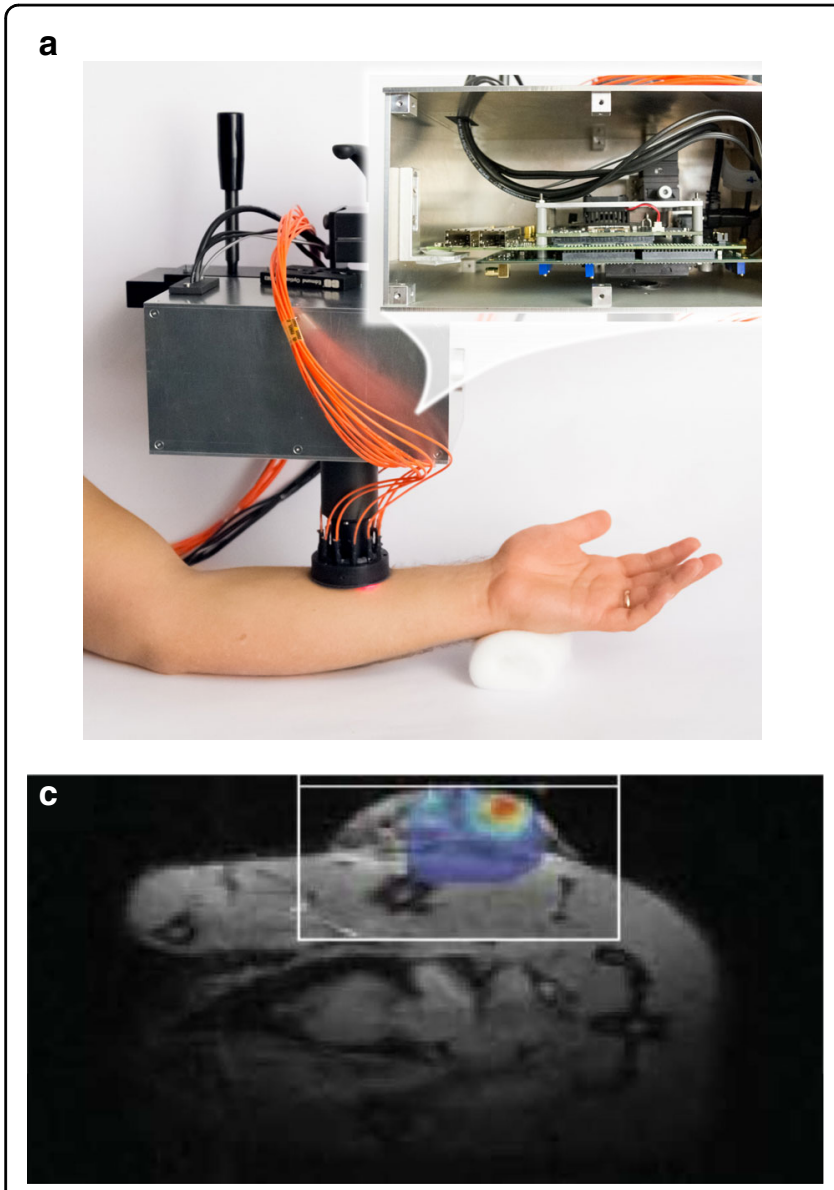

b
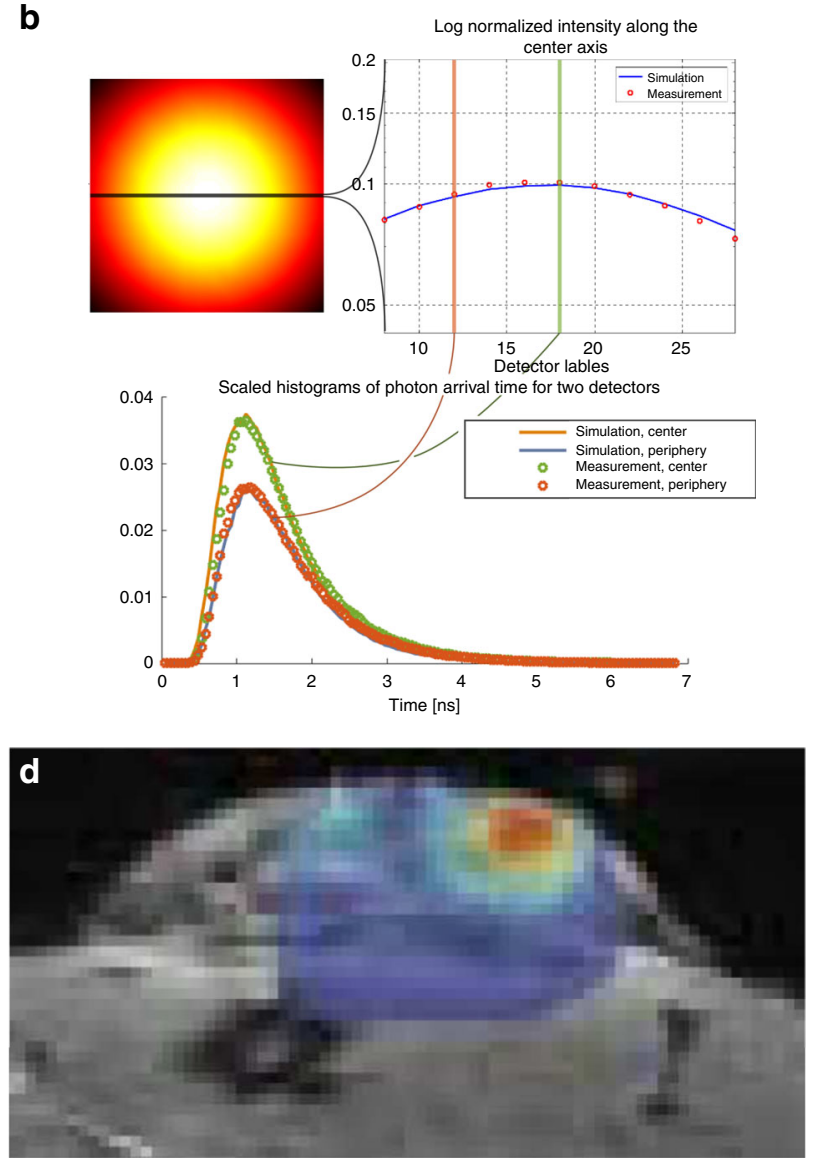

Fig. 6 SPAD optical tomography images and applications. a, b NIROT camera system prototype and measurements versus simulation results for a phantom ${ }^{154}$. c, d Fluorescence molecular tomography (FMT) image as an overlap of the optical image obtained with the RadHard2 $32 \times 32$ photoncounting sensor with the corresponding MRI image ${ }^{156}$. C51 cells (a colon cancer-derived cell line) have been implanted in the flank of a mouse. A clear spread in the protease activity, indicated by the significantly higher fluorescence intensity in some parts of the tumour, is shown. c Complete MR + FMT image, and $\mathbf{d}$ zoom of the cancer region. The images are reprinted from refs. ${ }^{154,156}$

FMT (fluorescence molecular tomography) relies on (exogenous) fluorescent molecules to enable the reconstruction of their concentration distribution in 3D; FMT can be applied to small animals for pre-clinical cancer research, and possibly over time to track dynamic phenomena. A hybrid MRI-FMT imaging system was demonstrated in refs. ${ }^{156,157}$, with the imaging component based on the RadHard2 (ref. ${ }^{107}$ ) $32 \times 32$ photon-counting SPAD array. The results are shown in Fig. 6, where the overlap of the MRI and FMT images of a mouse tumour is displayed in Fig. 6c and enlarged in Fig. 6d. The corresponding findings were later confirmed by histological analysis of the tumour.

Finally, it is worthwhile to mention the possibility of using the exquisite timing capabilities and spatial resolution of SPAD arrays to detect ballistic and snake photons that have travelled through tissue, such as demonstrated in ref. ${ }^{158}$. The MEGAFRAME32 sensor was employed to locate the distal-end of a fibre-optic device deep in tissue, with $\mathrm{cm}$ resolution, within clinically relevant settings and in chest and lung models on one hand, and through the entire thickness of a human torso on the other.

\section{Conclusions}

Recent, fully integrated SPAD imagers developed for NIROT show a substantial increase in the array resolution and photon throughput due to on-chip time-stamping and histogram generation, over and beyond what can be obtained by composing arrays of single devices or SiPMs. This will likely lead to improved spatial resolution in the reconstructed image, albeit at the expense of increased system complexity. Further developments focus on an increase in the sensitivity in the red and NIR regions on one hand, and an increase in the dynamic range by timedomain gating on the other. The latter will enable to measure faster and deeper in tissue (potentially $2 \times$ with respect to a non-gated scenario). 


\section{Other biophotonics applications and sensor concepts \\ PET}

Among several other biophotonics applications, which have been the subject of investigations with SPAD arrays, the nuclear medicine domain, and PET in particular, have a prominent role. Its peculiar architectural implications have already been analysed at the end of the Array architecture section, and are particularly interesting because they basically span the full pixel granularity range, from small (mm-sized) silicon photomultipliers (SiPMs) where all SPADs are connected together to provide a common output, to imagers with individually addressable SPAD pixels. The former, which are already employed in analogue form in top-end PET systems, are being revisited, e.g. monolithically with the addition of on-chip TDCs, while keeping backward compatibility ${ }^{159}$, or by coupling an off-theshelf analogue SiPM array to an FPGA-based board to enable advanced timing functionality with relatively simple hardware modifications ${ }^{160}$. The latter are being extended in 3D, as will be described in the corresponding section below.

\section{Q-LSRM}

On the more future-oriented side, the SPAD's spatial and time-resolved capabilities are being investigated to enable quantum-based super-resolution microscopy (QLSRM). In principle, using a quantum correlated $N$-photon state combined with an optical centroid measurement (OCM) allows a resolution enhancement of $1 / N$. Apart from the significant challenges on the source side, such an approach calls for a high detection efficiency, timing resolution and frame rates on the detection side, while minimising the crosstalk and DCR. An early implementation of a monolithic $4 \times 4 \mathrm{G}^{(2)}$ SPAD imager was reported in refs. ${ }^{161,162}$, which aimed at resolving secondorder intensity correlations. More recently, the SUPERTWIN project started looking at all solid-state technologies for the generation and recording of entangled photons, targeting a resolution of $40 \mathrm{~nm}$. The much larger and advanced SPADnet1 SPAD array, originally designed for PET applications, was employed in a first proof-ofprinciple experiment to detect spatially entangled photon pairs, generated by spontaneous parametric downconversion (SPDC) in a non-linear crystal pumped with an intense laser beam ${ }^{163,164}$. The collected experience from this approach allowed to proceed with the design of an ad hoc detector, incorporating on-chip features to increase the duty cycle (e.g. to avoid reading empty frames). A $32 \times 32$ SPAD array was manufactured in a $150 \mathrm{~nm}$ CMOS process, allowing $50 \mathrm{~ns}$ long observation windows at up to $800 \mathrm{kHz}$ to measure the 1 st $\left(G^{(1)}\right)$ and 2nd $\left(G^{(2)}\right)$ order correlation functions, and the array was experimentally characterised again with a source of entangled photon pairs ${ }^{36}$.

\section{Alternatives to monolithic approaches}

A sensor concept that is an alternative to the monolithic approaches described so far, built around the use of "reconfigurable pixels", was already hinted at the end of the "Read-out architecture" section. The underlying idea is to be able to reconfigure the main data processing features as a function of the specific application needs, as is the case with the LinoSPAD $256 \times 1$ linear array. This system is designed in such a way that the sensor layer hosting the actual SPADs (with a fill factor of 43\%) is decoupled from the data processing features, which are embedded in a companion FPGA. The latter contains core processing blocks such as a 64-element parallel TDC array $^{54,55}$ for time-correlated applications, whereas others are reconfigurable and can in principle be combined in a modular fashion. Another advantage of this approach resides in the possible combination of a sensing-optimised layer with a more advanced processing tier (e.g. 40 or $28 \mathrm{~nm}$ ) and the possibility of exchanging one or the other as technology evolves or new firmware is designed.

\section{D-stacking}

The combination of a top sensor layer with a bottom (likely all-digital CMOS) control and processing layer, each optimised for the respective function, can also be achieved with 3D-stacking techniques (see Fig. 7a for a concept image); these techniques are progressively becoming accessible to a larger user community and benefit from the developments in consumer markets (e.g. cameras for mobile phone applications), where significant resources are available. Such an approach could potentially enable a high PDE, low DCR and reduced jitter and afterpulsing, while adding advanced functionality and low power consumption due to the use of smaller technology nodes in the bottom-tier. In addition, the use of compound semiconductors for the top layer, such as InGaAs, could open up additional imaging windows in the NIR and even mid-wave IR regions, leading to enhanced tissue penetration (see, for example, Fig. $7 \mathrm{~d}$ and refs. ${ }^{165,166}$ for a summary of extensive work in this direction by MIT's Lincoln Laboratory). Another route to an enhanced NIR sensitivity with silicon-based platforms is backside illumination (BSI), similar to what has already been implemented in most high-end CMOS consumer imagers, whereby the substrate of the top wafer needs to be thinned down to only a few micrometres; this can be combined with thicker active volumes to counteract the reduced absorption of silicon in the NIR region. In general, the cost associated with moving to $3 \mathrm{D}$ is a higher design complexity, challenging 3D (wafer level) bonding techniques and the corresponding development costs. 

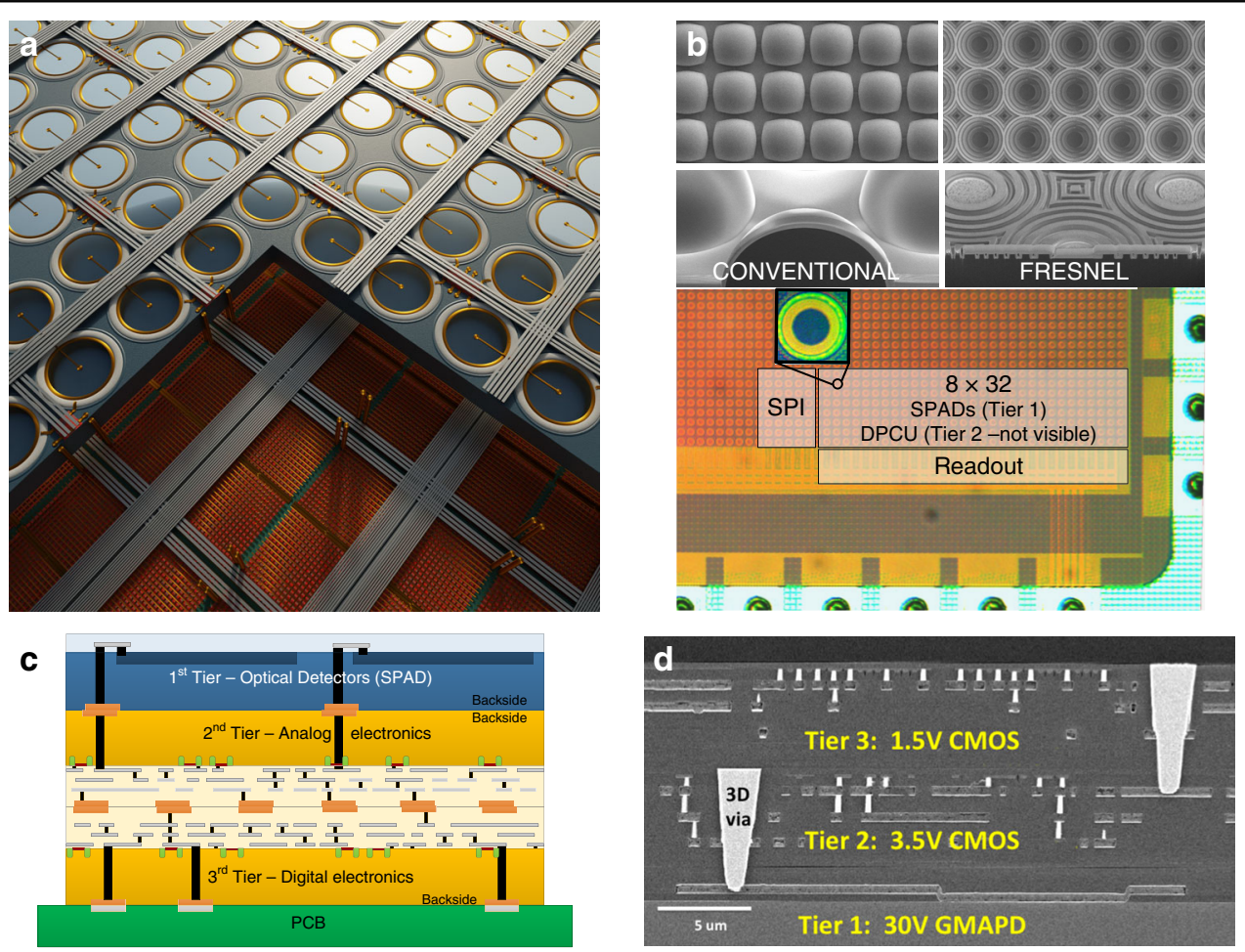

Fig. 7 Recent SPAD concepts for imagers revolve around 3D integration, possibly combined with microlenses to further maximise the fill factor. a A 3D integration concept image, $\mathbf{b}$ a two-tier implementation with additional microlenses ${ }^{179}$ and $\mathbf{c}$, $\mathbf{d}$ cross-sections of different imagers using three tiers ${ }^{165,172}$. Frontside illumination is used in $\mathbf{c}$, whereas backside illumination is used in $\mathbf{b}$ and $\mathbf{d}$. The images $\mathbf{b}$-d are reprinted from refs. $165,172,179$

Early work towards CMOS 3D IC SPAD-based imagers included a proof-of-concept $400 \times 1$ linear array specifically designed with NIROT applications in mind ${ }^{152,167}$, which combined 400 SPADs on the top tier with 100 TDCs ( $50 \mathrm{ps} \mathrm{LSB}$ ) on the bottom layer in a $130 \mathrm{~nm}$ process. In parallel, the use of silicon-on-insulator (SOI) was explored in refs. ${ }^{168,169}$, with the aim of fabricating an array of $32 \times 32$ custom (BackSPAD) photodetectors in a $3-\mu \mathrm{m}$-thick SOI film of an SOI wafer $(0.35-\mu \mathrm{m}$ feature size). The latter was then flipped and wafer bonded to a second $0.35 \mu \mathrm{m}$ CMOS wafer with the ancillary electronics. A large body of work is available from Sherbrooke on 3D approaches specifically dedicated to PET, with the aim of achieving a possible "ultimate" one-to-one coupling between each pixel and its corresponding quenching and timing electronics ${ }^{170,171}$. An important part of the effort was dedicated, not surprisingly, to the 3D integration process itself, with proofs of principle along the way such as $^{172}$ detailing the design of the top tier $0.8-\mu \mathrm{m}$ highvoltage CMOS SPADs in a frontside illumination approach (FSI, see Fig. 7c), and the corresponding challenges in terms of connections (use of through silicon vias).

More recently, several papers have reported work exploiting advanced commercially available 3D BSI technologies. The work was in part driven by the need for optimised, small-pitch SPAD arrays with enhanced red and NIR sensitivity for consumer applications, such as light detection and ranging (LiDAR); this has led to SPAD implementations that were unthinkable in such technology nodes just a few years ago, opening the door to a potential extension to megapixel arrays. In ref. ${ }^{173}$, for example, the pixel pitch was reduced to $7.83 \mu \mathrm{m}$, leading to a $128 \times 120$ time-gated prototype combining an imaging specific $65 \mathrm{~nm}$ top tier with a $40 \mathrm{~nm}$ bottom-tier employing 1-to-1 hybrid bond connections. A different implementation in the same 3D IC CMOS process was reported in $^{174}$, achieving a higher PDE due to cascoded passive quenching and active recharging, while still being compatible with the transistor operating voltage regimes of such highly scaled technologies. The example in Fig. 7b employs a $45 \mathrm{~nm}$ sensor tier and a $65 \mathrm{~nm}$ low-power processing tier, adding microlenses for fill factor enhancement. Very low afterpulsing was obtained with a very short $8 \mathrm{~ns}$ dead time. Finally, the top tier was further scaled down to $45 \mathrm{~nm}$ in ref. ${ }^{175}$, while further improving the peak PDP and reducing the jitter and DCR. The DCR is in general 2-3 orders of magnitude higher than the best FSI technologies, whereas the PDP, which is typically very low in the blue region for BSI implementations, has 


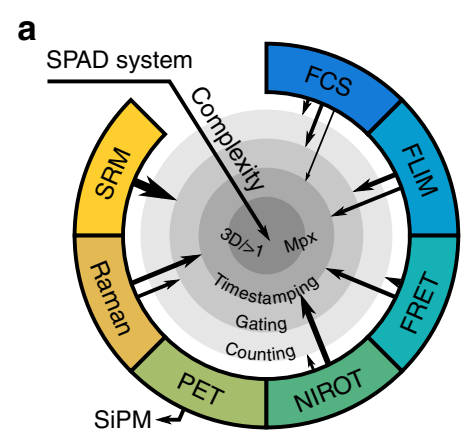

C
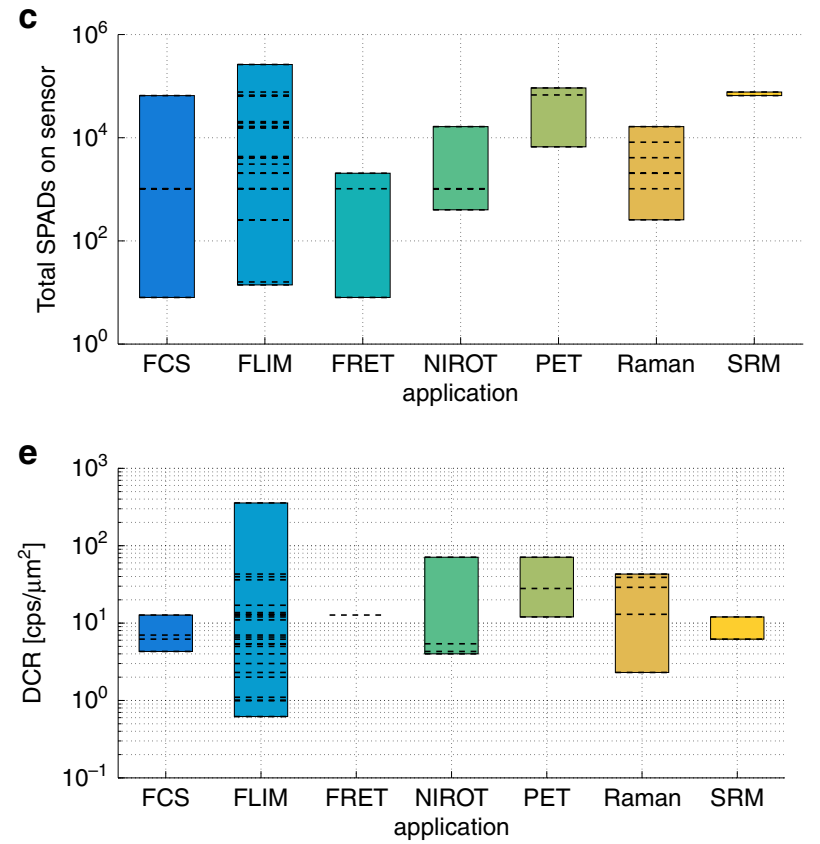

b

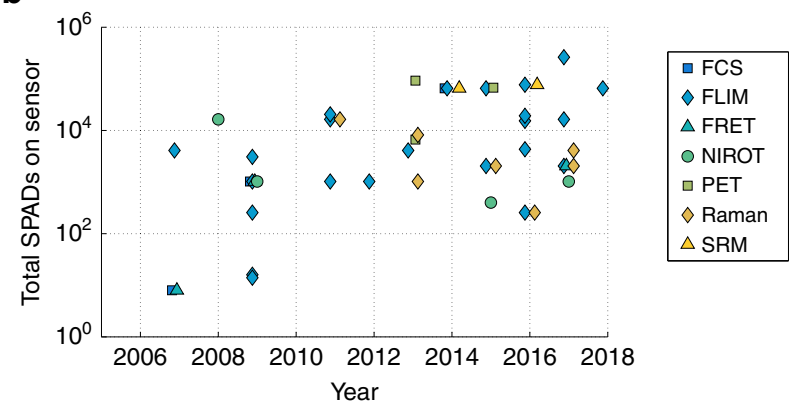

d

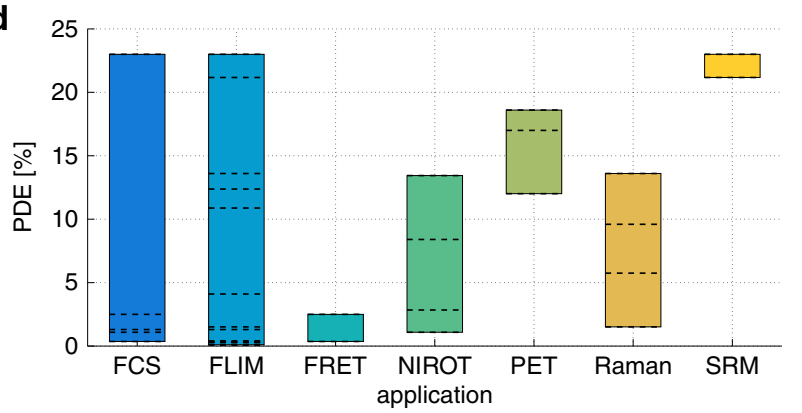

f

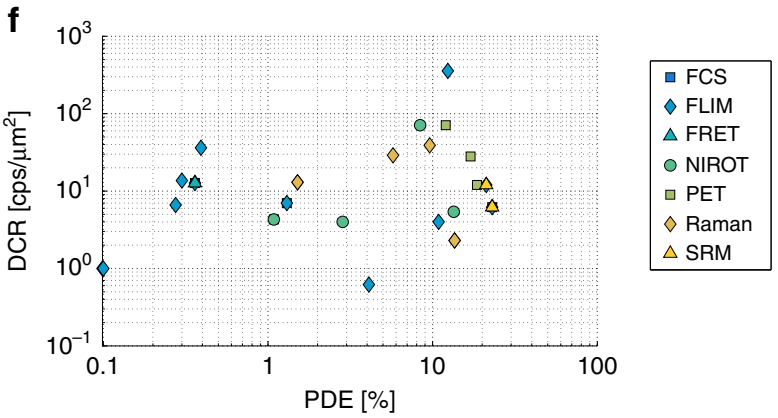

Fig. 8 SPAD system complexity vs. biophotonics applications and evolution of representative SPAD sensor figures of merit. a Schematic overview of the SPAD-based system complexity, in terms of key functionalities (counting/gating/time-stamping) versus the main biophotonics applications. b-f Overview of the representative SPAD sensor figures of merit as a function of the main target applications, based on data from Table 2: b total number of SPADs (corresponding to the effective spatial resolution in the imagers) versus time; $\mathbf{c}-\mathbf{e}$ total number of SPADs, PDE and DCR per unit area grouped based on the application types (dashed lines: individual sensors, top/bottom of each box: maximum/minimum); and $\mathbf{f}$ the DCR per unit area versus the PDE

improved to above $30 \%$ in the red region, with fill factors in excess of $50 \%$.

\section{Summary}

Figure 8a provides a schematic overview of how the key functionalities of the SPAD-based imagers reviewed in this paper are related to the main biophotonics applications to which they have been applied so far. The arrows pointing towards the centre provide a qualitative indication of the relative importance of the different functionalities, whereby most applications have seen the use of at least two of the functionalities (e.g. time-stamping and gating), except localisation-based super-resolution microscopy (SRM), which has only needed photon counting so far. PET is a somewhat special case, relying on ad hoc architectures as discussed in the first paragraph of the previous section.

Figure 8b shows the distribution of the total number of SPADs over time, based on the data from Table 2, indicating a clear trend towards larger SPAD arrays over the past decade. Figure $8 \mathrm{c}-\mathrm{e}$ illustrates the distribution of some of the key figures of merit (total number of SPADs, PDE and DCR per unit area), again using Table 2 as a reference, grouped based on the main biophotonics application types. Each dashed line corresponds to an individual sensor. For the total number of SPADs and the PDE, higher values are preferred, whereas the contrary is obviously true for the DCR. The size of each box is 
representative of the spread of the figures of merit for that specific application; the size is, for example, larger for FLIM, where the first developments took place early on, when the technology and designs were far from optimised. The box size also reflects the number of sensors that have been employed thus far; from this perspective, the size is smaller for SRM, which has only been addressed very recently.

Finally, as discussed in the SPAD section, the design of SPAD sensors entails numerous trade-offs, such as the PDE versus DCR shown in Fig. 8f. This plot indicates a move towards higher PDE values, although this is not easy to achieve while still maintaining a low DCR (an ideal sensor would be placed in the bottom right corner of this plot).

\section{Conclusions and outlook}

In this review, we have focused on SPAD imagers in standard CMOS technologies and their biophotonics applications. Individual SPAD pixels and small arrays have evolved from a scientific curiosity 15 years ago to a range of fully integrated devices, where the key challenges have been the sensitivity, homogeneity, noise, reliability and reproducibility.

At the device level, the performance gap between SPADs fabricated in custom technologies and in standard CMOS technology has been shrinking over time, whereby CMOS can leverage 60 years of experience and investments in scalable and high-yield technologies. The corresponding improvements have been significant in terms of key parameters, such as the PDP and fill factor, with peak values in excess of $40-55 \%$, the DCR, which has been drastically reduced to below $0.1 \mathrm{cps} / \mu \mathrm{m}^{2}$ in the best SPADs, and the percentage of hot pixels (just a few percent in the best technologies). The spatial resolution has also increased to a quarter megapixel for the largest formats, with megapixel arrays on the horizon. This has called for a decrease of the pixel size to well below $10 \mu \mathrm{m}$, with some groups targeting SPADs in deep sub-micron technology nodes ( $45 \mathrm{~nm}$ and below), not very far from their counterparts in CMOS imagers; this feat was unthinkable just a few years ago. However, achieving multi-megapixel SPAD sensors is challenging, as SPADs cannot be easily decreased in size below $3 \mu \mathrm{m}$ (pitch) in currently available technologies (see ref. ${ }^{176}$ for results on a $4 \times 4$ test structure). This is due to the required guard ring and the correlated reduction of the active area to zero due to edge effects. The timing accuracy was already excellent (typically $50-100 \mathrm{ps}$, with the best SPADs in the 20-30 ps range) and has, therefore, evolved in a less spectacular fashion.

At the architectural level, standard CMOS SPAD arrays have a clear advantage over CCDs/sCMOS imagers, when parallel single-photon counting and/or timing is required; standard CMOS SPAD arrays can be coupled in a flexible way to different digital blocks for data acquisition and/or processing in close proximity to the sensor, thanks to the natively digital SPAD data output. The absence of readout noise is an important issue that is often neglected, particularly when SPAD imagers are coupled to very high speed ( 100 kfps), ADC-less binary implementations, delivering continuous microsecond frames in real-time to capture fast transient phenomena. The capability of implementing an integrated, parallel nanosecond gating is also a very appealing alternative to non-all-solid-state implementations. However, such short gate windows have to be properly implemented to achieve a reasonable skew (below $150 \mathrm{ps}$ ) and with the corresponding steep rise and fall times. This can be challenging in large SPAD arrays due to the power consumption required by the distribution of the digital gating signals across the array.

Therefore, it is not surprising that FLIM has been explored first, together with multibeam approaches that make full use of the sensors' native parallelism. This was then followed by a host of other time-resolved biophotonics applications, all the way to disruptive scenarios, such as quantum-based super-resolution microscopy; the latter represents a good example of a sensor architecture that has been custom-designed to meet specific requirements, for which efficient sensor alternatives are rare. It is, however, true that most SPAD imagers are still research prototypes, and only some are available commercially, e.g. from SMEs such as PhotonForce (UK) or MPD (Italy), usually as a spin-off of designs explored in academic environments. (Other commercially available SPAD arrays are usually derived from non-imaging SiPMs, e.g. the Philips Digital Photon Counting dSiPM for clinical PET, or the STMicroelectronics time-of-flight sensors ${ }^{177}$, aimed at ranging in the consumer market).

This is partly due to the maturity of the sensors themselves and/or of the underlying technology, and partly to the fact that the overall sensitivity, in particular in the red region, still lags behind the sensitivity of $\mathrm{CCD} / \mathrm{sCMOS}$ imagers by $2-10 x$. This can be an issue in applications where absolute sensitivity, rather than the SNR, is important, or where the illumination power needs to be kept low to avoid sample degradation. Certain applications require a very high timing resolution, which is typical of single, highly optimised SPADs, and can tolerate longer acquisition times due to scanning. It is also true that for SPAD imagers, system integration can typically be more complex due to the high data rates and corresponding tight interconnect and firmware requirements. Finally, in certain cases SPAD designers face competition from other single-photon or established technologies.

In the future, we foresee that academic and research establishments will continue pushing the state-of-the-art in terms of the key figures of merit, targeting large-format 
and high-performance CMOS SPAD arrays, with industrial applications starting to appear in the medium term. Larger industrial conglomerates are more likely to emphasize high-volume applications in the mobile/consumer areas, e.g covering automotive (in particular LiDAR for advanced driver-assistance systems), point-of-care and Internet of Things (IoT) applications, possibly via smaller, dedicated units; these foundries might also propose SPADs as IP blocks in the not too distant future. The resulting consumer oriented developments are likely to follow trends similar to those that characterised high-end smartphone imagers (i.e. towards chip stacking and 3D ICs); these developments might impact in a positive way niche markets in general, and time-resolved applications of interest to the biophotonics community in particular.

\section{Acknowledgements}

This work was supported in part by the Swiss National Science Foundation Grant 166289. The authors would like to thank Scott Lindner and Arin Can Ulku (EPFL AQUA) for their kind assistance.

\section{Conflict of interest}

The authors declare that they have no conflict of interest.

Received: 28 November 2018 Revised: 30 July 2019 Accepted: 7 August 2019

Published online: 18 September 2019

\section{References}

1. Zappa, F., Tisa, S., Tosi, A. \& Cova, S. Principles and features of single-photon avalanche diode arrays. Sens. Actuat. A 140, 103-112 (2007).

2. Zappa, F., Tosi, A., Dalla Mora, A., Guerrieri, F. \& Tisa, S. Single-photon avalanche diode arrays and CMOS microelectronics for counting, timing, and imaging quantum events. In Proc. SPIE, Quantum Sensing and Nanophotonic Devices VII, $76082 C$ (SPIE, San Francisco, CA, United States, 2010).

3. Charbon, E. Single-photon imaging in complementary metal oxide semiconductor processes. Philos. Trans. R. Soc. Lond. Ser. A 372, 20130100 (2014).

4. Perenzoni, M., Pancheri, L. \& Stoppa, D. Compact SPAD-based pixel architectures for time-resolved image sensors. Sensors 16, 745 (2016).

5. Rochas, A. et al. Single photon detector fabricated in a complementary metal-oxide-semiconductor high-voltage technology. Rev. Sci. Instrum. 74, 3263-3270 (2003).

6. Rochas, A. et al. First fully integrated 2-D array of single-photon detectors in standard CMOS technology. IEEE Photonics Technol. Lett. 15, $963-965$ (2003).

7. Bronzi, D., Villa, F., Tisa, S., Tosi, A. \& Zappa, F. SPAD figures of merit for photon-counting, photon-timing, and imaging applications: a review. IEEE Sens. J. 16, 3-12 (2016)

8. Esposito, A. Beyond range: innovating fluorescence microscopy. Remote Sens. 4. 111-119 (2012)

9. Henderson, R. K., Rae, B. R. \& Li, D.-U. Complementary Metal-OxideSemiconductor (CMOS) Sensors for Fluorescence Lifetime Imaging (FLMM), Ch. 11, 312-347 (Elsevier, 2014).

10. Suhling, $K$. et al. Fluorescence lifetime imaging (FLIM): basic concepts and some recent developments. Med. Photonics 27, 3-40 (2015).

11. Caccia, M., Nardo, L., Santoro, R. \& Schaffhauser, D. Silicon photomultipliers and SPAD imagers in biophotonics: advances and perspectives. Nucl. Instrum. Methods Phys. Res. Sect. A 926, 101-117 (2019).

12. Niclass, C., Rochas, A., Besse, P.-A. \& Charbon, E. Design and characterization of a CMOS 3-D image sensor based on single photon avalanche diodes. IEEE J. Solid-State Circuits 40, 1847-1854 (2005).

13. Webster, E. A., Grant, L. A. \& Henderson, R. K. A high-performance singlephoton avalanche diode in 130-nm CMOS imaging technology. IEEE Electron Device Lett. 33, 1589-1591 (2012).
14. Fishburn, M. W., Maruyama, Y. \& Charbon, E. Reduction of fixed-position noise in position-sensitive single-photon avalanche diodes. IEEE Trans. Electron Devices 58, 2354-2361 (2011).

15. Burri, $\mathrm{S}$. et al. Architecture and applications of a high resolution gated SPAD image sensor. Opt. Express 22, 17573-17589 (2014).

16. Eisele, A. et al. $185 \mathrm{MHz}$ count rate $139 \mathrm{~dB}$ dynamic range single-photon avalanche diode with active quenching circuit in $130 \mathrm{~nm}$ CMOS technology. In Proc. IISW 278-280 (IISW, Hokkaido, Japan, 2011).

17. Niclass, C., Favi, C., Kluter, T., Gersbach, M. \& Charbon, E. A $128 \times 128$ singlephoton image sensor with column-level 10-bit time-to-digital converter array. IEEE J. Solid-State Circuits 43, 2977-2989 (2008).

18. Pancheri, L. \& Stoppa, D. A SPAD-based pixel linear array for high-speed timegated fluorescence lifetime imaging. In Proc. ESSCIRC 428-431(IEEE, Athens, Greece, 2009).

19. Stoppa, D., Mosconi, D., Pancheri, L. \& Gonzo, L. Single-photon avalanche diode CMOS sensor for time-resolved fluorescence measurements. IEEE Sens. J. 9, 1084-1090 (2009).

20. Maruyama, Y. \& Charbon, E. An all-digital, time-gated $128 \times 128$ SPAD array for on-chip, filter-less fluorescence detection. In Solid-State Sensors Actuators and Microsystems Conference 1180-1183 (IEEE, Beijing, China, 2011).

21. Gersbach, M. et al. A time-resolved, low-noise single-photon image sensor fabricated in deep-submicron CMOS technology. IEEE J. Solid-State Circuits 47, 1394-1407 (2012).

22. Mandai, S. \& Charbon, E. A $4 \times 4 \times 416$ digital SiPM array with 192 TDCs for multiple high-resolution timestamp acquisition. J. Instrum. 8, P05024 (2013).

23. Carimatto, A. et al. A 67,392-SPAD PVTB-compensated multi-channel digital SiPM with 432 column-parallel 48 ps 17b TDCs for endoscopic time-of-flight PET. In IEEE ISSCC Digest of Technical Paper 1-3 (IEEE, San Francisco, CA, United States, 2015).

24. Krstajić, N., Levitt, J., Poland, S., Ameer-Beg, S. \& Henderson, R. $256 \times 2$ SPAD line sensor for time resolved fluorescence spectroscopy. Opt. Express 23, 5653-5669 (2015).

25. Dutton, N. A. et al. A SPAD-based QVGA image sensor for single-photon counting and quanta imaging. IEEE Trans. Electron Devices 63, 189-196 (2016).

26. Homulle, H. et al. Compact solid-state CMOS single-photon detector array for in vivo NIR fluorescence lifetime oncology measurements. Biomed. Opt. Express 7, 1797-1814 (2016).

27. Kröger, J. et al. High intensity click statistics from a $10 \times 10$ avalanche photodiode array. J. Phys. B Mol. Opt. Phys. 50, 214003 (2017).

28. Antolovic, I. M., Bruschini, C. \& Charbon, E. Dynamic range extension for photon counting arrays. Opt. Express 26, 22234-22248 (2018).

29. Ulku, A. C., Bruschini, C., Michalet, X., Weiss, S. \& Charbon, E. A 512×512 SPAD image sensor with built-in gating for phasor based real-time siFLIM. In Proc. IISW 234-237 (IISW, Hiroshima, Japan, 2017).

30. Gyongy, l. et al. A $256 \times 256,100 \mathrm{kfps}, 61 \%$ fill-factor SPAD image sensor for time-resolved microscopy applications. IEEE Trans. Electron Devices 65, 547-554 (2018).

31. Niclass, C., Favi, C., Kluter, T., Monnier, F. \& Charbon, E. Single-photon synchronous detection. IEEE J. Solid-State Circuits 44, 1977-1989 (2009).

32. Lee, C., Johnson, B., Jung, T. \& Molnar, A. A $72 \times 60$ angle-sensitive SPAD imaging array for lens-less FLIM. Sensors 16, 1422 (2016).

33. Veerappan, C. et al. A $160 \times 128$ single-photon image sensor with on-pixel 55 ps 10b time-to-digital converter. In IEEE ISSCC Digest in Technical Paper 312-314 (IEEE, San Francisco, CA, United States, 2011).

34. Field, R. M. \& Shepard, K. A 100 fps fluorescence lifetime imager in standard $0.13 \mu \mathrm{m}$ CMOS. In IEEE Symposium on VLSI Circuits C10-C11 (IEEE, Kyoto, Japan, 2013).

35. Field, R. M., Realov, S. \& Shepard, K. L. A 100 fps, time-correlated singlephoton-counting-based fluorescence-lifetime imager in $130 \mathrm{~nm}$ CMOS. IEEE J. Solid-State Circuits 49, 867-880 (2014).

36. Gasparini, L. et al. A $32 \times 32$ pixel time-resolved single-photon image sensor with $44.64 \mu \mathrm{m}$ pitch and $19.48 \%$ fill-factor with on-chip row/frame skipping features reaching $800 \mathrm{kHz}$ observation rate for quantum physics applications. In IEEE ISSCC Digest in Technical Paper 98-100 (IEEE, San Francisco, CA, United States, 2018).

37. Parmesan, L. et al. A $256 \times 256$ SPAD array with in-pixel time to amplitude conversion for fluorescence lifetime imaging microscopy. In Proc. IISW 9.04 (IISW, Vaals, Netherlands, 2015).

38. Perenzoni, M., Massari, N., Perenzoni, D., Gasparini, L. \& Stoppa, D. A $160 \times 120$ pixel analog-counting single-photon imager with time-gating and self- 
referenced column-parallel A/D conversion for fluorescence lifetime imaging. IEEE J. Solid-State Circuits 51, 155-167 (2016).

39. Donati, S., Martini, G. \& Norgia, M. Microconcentrators to recover fill-factor in image photodetectors with pixel on-board processing circuits. Opt. Express 15, 18066-18075 (2007).

40. Donati, S., Martini, G. \& Randone, E. Improving photodetector performance by means of microoptics concentrators. J. Light. Technol. 29, 661-665 (2011).

41. Pavia, J. M., Wolf, M. \& Charbon, E. Measurement and modeling of microlenses fabricated on single-photon avalanche diode arrays for fill factor recovery. Opt. Express 22, 4202-4213 (2014).

42. Intermite, G. et al. Enhancing the fill-factor of CMOS SPAD arrays using microlens integration. In Proc. SPIE, Photon Counting Applications, 95040J (SPIE, Prague, Czech Republic, 2015).

43. Intermite, G. et al. Fill-factor improvement of Si CMOS single-photon avalanche diode detector arrays by integration of diffractive microlens arrays. Opt. Express 23, 33777-33791 (2015).

44. Antolovic, I. M., Burri, S., Bruschini, C., Hoebe, R. \& Charbon, E. Nonuniformity analysis of a 65 kpixel CMOS SPAD imager. IEEE Trans. Electron Devices 63, 57-64 (2016).

45. Gyongy, I. et al. Cylindrical microlensing for enhanced collection efficiency of small pixel SPAD arrays in single-molecule localisation microscopy. Opt. Express 26, 2280-2291 (2018).

46. Zhang, C., Lindner, S., Antolovic, l., Wolf, M. \& Charbon, E. A CMOS SPAD imager with collision detection and 128 dynamically reallocating TDCs for single-photon counting and 3D time-of-flight imaging. Sensors 18, 4016 (2018).

47. Schaart, D. R., Charbon, E., Frach, T. \& Schulz, V. Advances in digital SiPMs and their application in biomedical imaging. Nucl. Instrum. Methods Phys. Res. Sect. A 809, 31-52 (2016)

48. Walker, R. J. et al. A 92k SPAD time-resolved sensor in $0.13 \mu \mathrm{m}$ CIS technology for PET/MRI applications. In Proc. IISW 1-4 (IISW, Snowbird, UT, United States, 2013).

49. Braga, L. H. et al. A fully digital $8 \times 16$ SiPM array for PET applications with per-pixel TDCs and real-time energy output. IEEE J. Solid-State Circuits 49, 301-314 (2014).

50. Li, D.-U. et al. Real-time fluorescence lifetime imaging system with a $32 \times 32$ $0.13 \mu \mathrm{m}$ CMOS low dark-count single-photon avalanche diode array. Opt. Express 18, 10257-10269 (2010).

51. Li, D.-U. et al. Video-rate fluorescence lifetime imaging camera with CMOS single-photon avalanche diode arrays and high-speed imaging algorithm. J. Biomed. Opt. 16, 096012-096012 (2011).

52. Li, D. \& Chen, Y. Hardware-friendly bi-exponential fluorescence lifetime imaging algorithms and phasor approaches. In Proc. SPIE, Advanced Microscopy Techniques N; and Neurophotonics III, 95360M (SPIE, Munich, Germany, 2015).

53. Buchholz, J. et al. FPGA implementation of a $32 \times 32$ auto-correlator array for analysis of fast image series. Opt. Express 20, 17767-17782 (2012).

54. Burri, S., Bruschini, C. \& Charbon, E. LinoSPAD: a compact linear SPAD camera system with 64 FPGA-based TDC modules for versatile 50 ps resolution timeresolved imaging. Instruments 1, 6.1-6.21 (2017).

55. Burri, S., Homulle, H., Bruschini, C. \& Charbon, E. LinoSPAD: a time-resolved $256 \times 1$ CMOS SPAD line sensor system featuring 64 FPGA-based TDC channels running at up to 8.5 giga-events per second. In Proc. SPIE, Optical Sensing and Detection N, 98990D (SPIE, Brussels, Belgium, 2016).

56. Becker, W. Fluorescence lifetime imaging - techniques and applications. J. Microsc. 247, 119-136 (2012).

57. Lakowicz, J. R., Szmacinski, H., Nowaczyk, K., Berndt, K. W. \& Johnson, M. Fluorescence lifetime imaging. Anal. Biochem. 202, 316-330 (1992).

58. Marcu, L. Fluorescence lifetime techniques in medical applications. Ann. Biomed. Eng. 40, 304-331 (2012).

59. Hirvonen, L. M. \& Suhling, K. Wide-field TCSPC: methods and applications. Meas. Sci. Technol. 28, 012003 (2016).

60. Stegehuis, P. L. et al. Fluorescence lifetime imaging to differentiate bound from unbound ICG-CRGD both in vitro and in vivo. In Proc. SPIE, Advanced Biomedical and Clinical Diagnostic and Surgical Guidance Systems XIII, 931300 (SPIE, San Francisco, CA, United States, 2015).

61. Léonard, J. et al. High-throughput time-correlated single photon counting. Lab a Chip 14, 4338-4343 (2014).

62. Arlt, J. et al. A study of pile-up in integrated time-correlated single photon counting systems. Rev. Sci. Instrum. 84, 103105 (2013).

63. Tyndall, D. et al. A 100 Mphoton/s time-resolved mini-silicon photomultiplier with on-chip fluorescence lifetime estimation in $0.13 \mu \mathrm{m}$ CMOS imaging technology. In IEEE ISSCC Digest in Technical Paper 122-124 (IEEE, San Francisco, CA, United States, 2012).

64. Tyndall, D. et al. A high-throughput time-resolved mini-silicon photomultiplier with embedded fluorescence lifetime estimation in $0.13 \mu \mathrm{m}$ CMOS. IEEE Trans. Biomed. Circuits Syst. 6, 562-570 (2012).

65. Popleteeva, M. et al. Fast and simple spectral FLIM for biochemical and medical imaging. Opt. Express 23, 23511-23525 (2015).

66. Hanley, Q. S., Arndt-Jovin, D. J. \& Jovin, T. M. Spectrally resolved fluorescence lifetime imaging microscopy. Appl. Spectrosc. 56, 155-166 (2002).

67. Ehrlich, K. et al. Fibre optic time-resolved spectroscopy using CMOS-SPAD arrays. In Proc. SPIE, Optical Fibers and Sensors for Medical Diagnostics and Treatment Applications XVII, 10058 (SPIE, San Francisco, CA, United States, 2017).

68. Kufcsák, A. et al. Time-resolved spectroscopy at 19,000 lines per second using a CMOS SPAD line array enables advanced biophotonics applications. Opt. Express 25, 11103-11123 (2017)

69. Erdogan, A. T. et al. A 16.5 giga events/s $1024 \times 8$ SPAD line sensor with perpixel zoomable 50 ps-6.4 ns/bin histogramming TDC. In IEEE Symposium in VLSI Circuits C292-C293 (IEEE, Kyoto, Japan, 2017).

70. Peronio, P. et al. 32 channel time-correlated-single-photon-counting system for high-throughput lifetime imaging. Rev. Sci. Instrum. 88, 083704 (2017).

71. Tsikouras, A. et al. Characterization of SPAD array for multifocal high-content screening applications. Photonics 3, 56 (2016).

72. Burri, S. Challenges and Solutions to Next-Generation Single-Photon Imagers. Ph.D. thesis, EPFL, Lausanne, 2016

73. Ulku, A. C. et al. A $512 \times 512$ SPAD image sensor with integrated gating for widefield FLIM. IEEE J. Sel. Top. Quantum Electron. 25, 1-12 (2019).

74. Ulku, A. C. et al. Phasor-based widefield FLIM using a gated $512 \times 512$ singlephoton SPAD imager. In Proc. SPIE, Multiphoton Microscopy in the Biomedical Sciences XIX, 10882M (SPIE, San Francisco, CA, United States, 2019).

75. Wargocki, P. M. et al. Imaging free and bound NADH towards cancer tissue detection using FLIM system based on SPAD array. In CLEO/Europe-EQEC 1-1 (IEEE, Munich, Germany, 2017).

76. Pancheri, L., Massari, N., Borghetti, F. \& Stoppa, D. A 32×32 SPAD pixel array with nanosecond gating and analog readout. In Proc. IISW 1-4 (IISW, Hokkaido, Japan, 2011).

77. Pancheri, L. et al. Protein detection system based on $32 \times 32$ SPAD pixel array. In Proc. SPIE, Optical Sensing and Detection III, 843913 (SPIE, Brussels, Belgium, 2012).

78. Pancheri, L., Massari, N. \& Stoppa, D. SPAD image sensor with analog counting pixel for time-resolved fluorescence detection. IEEE Trans. Electron Devices 60, 3442-3449 (2013).

79. Schwartz, D. E., Charbon, E. \& Shepard, K. L. A single-photon avalanche diode imager for fluorescence lifetime applications. In IEEE Symposium in VLSI Circuits 144-145 (IEEE, Kyoto, Japan, 2007).

80. Schwartz, D. E., Charbon, E. \& Shepard, K. L. A single-photon avalanche diode array for fluorescence lifetime imaging microscopy. IEEE J. Solid-State Circuits 43, 2546-2557 (2008).

81. Richardson, J. et al. A $32 \times 3250$ ps resolution 10-bit time to digital converter array in $130 \mathrm{~nm}$ CMOS for time correlated imaging. In IEEE Custom Integrated Circuits 77-80 (IEEE, Rome, Italy, 2009).

82. Krstajić, N. et al. 0.5 billion events per second time correlated single photon counting using CMOS SPAD arrays. Opt. Lett. 40, 4305-4308 (2015).

83. Veerappan, C. et al. Characterization of large-scale non-uniformities in a 20k TDC/SPAD array integrated in a $130 \mathrm{~nm}$ CMOS process. In Proc. ESSDERC 331-334 (IEEE, Helsinki, Finland, 2011).

84. Arlt, J. et al. A fully-integrated, time-resolved $160 \times 128$ SPAD pixel array with micro-concentrators. In Proc. Advanced Photon Counting Techniques V, SPIE Defense and Security (SPIE, Orlando, FL, United States, 2011).

85. Gersbach, M. et al. High frame-rate TCSPC-FLIM using a novel SPAD-based image sensor. In Proc. SPIE, Detectors and Imaging Devices: Infrared, Focal Plane, Single Photon, $77801 \mathrm{H}$ (SPIE, San Diego, CA, United States, 2010).

86. Li, D.-U. et al. Time-domain fluorescence lifetime imaging techniques suitable for solid-state imaging sensor arrays. Sensors 12, 5650-5669 (2012).

87. Giraud, G. et al. Fluorescence lifetime biosensing with DNA microarrays and a CMOS-SPAD imager. Biomed. Opt. Express 1, 1302-1308 (2010).

88. Coelho, S. et al. Multifocal multiphoton microscopy with adaptive optical correction. In Proc. SPIE, Multiphoton Microscopy in the Biomedical Sciences XIIII, 858817 (SPIE, San Francisco, CA, United States, 2013).

89. Poland, S. P. et al. Development of a fast TCSPC FLIM-FRET imaging system. In Proc. SPIE, Multiphoton Microscopy in the Biomedical Sciences XII, 8588 (SPIE, San Francisco, CA, United States, 2013). 
90. Poland, S. P. et al. Time-resolved multifocal multiphoton microscope for high speed FRET imaging in vivo. Opt. Lett. 39, 6013-6016 (2014).

91. Poland, S. P. et al. A high speed multifocal multiphoton fluorescence lifetime imaging microscope for live-cell FRET imaging. Biomed. Opt. Express 6, 277-296 (2015).

92. Poland, S. P. et al. New high-speed centre of mass method incorporating background subtraction for accurate determination of fluorescence lifetime. Opt. Express 24, 6899-6915 (2016).

93. Vitali, M. et al. A single-photon avalanche camera for fluorescence lifetime imaging microscopy and correlation spectroscopy. IEEE J. Sel. Top. Quantum Electron. 20, 344-353 (2014).

94. Clegg, R. M. Fluorescence resonance energy transfer. Curr. Opin. Biotechnol. 6 103-110 (1995).

95. Lindner, S. et al. A novel $32 \times 32,224$ Mevents/s time resolved SPAD image sensor for near-infrared optical tomography. In Optics and the Brain JTh5A-JTh56 (Optical Society of America, Hollywood, FL, United States, 2018).

96. Zhang, C. et al. A 30-frames/s, $252 \times 144$ SPAD flash LiDAR with 1728 dualclock 48.8-ps TDCs, and pixel-wise integrated histogramming. IEEE J. SolidState Circuits 54, 1137-1151 (2019).

97. Draaijer, A., Sanders, R. \& Gerritsen, H. in Handbook of Biological Confocal Microscopy (ed. Pawley, J. B.) 491-505 (Springer, Boston, MA, United States, 1995).

98. Gyongy, I. et al. Fluorescence lifetime imaging of high-speed particles with single-photon image sensors. In Proc. SPIE, High-Speed Biomedical Imaging and Spectroscopy IV, 1088900 (SPIE, San Francisco, CA, United States, 2019).

99. Ardelean, A., Ulku, A. C., Michalet, X., Charbon, E. \& Bruschini, C. Fluorescence lifetime imaging with a single-photon SPAD array using long overlapping gates: an experimental and theoretical study. In Proc. SPIE, Multiphoton Microscopy in the Biomedical Sciences XIX, 108820Y (SPIE, San Francisco, CA, United States, 2019).

100. Thompson, N. L. in Topics in Fluorescence Spectroscopy (ed. Lakowicz, J. R.) 337-378 (Springer, Boston, Massachusetts, United States, 2002).

101. Gösch, M. et al. Parallel single molecule detection with a fully integrated single-photon 2 × 2 CMOS detector array. J. Biomed. Opt. 9, 913-921 (2004).

102. Colyer, R. A. et al. Ultra high-throughput single molecule spectroscopy with a 1024 pixel SPAD. In Proc. SPIE, Single Molecule Spectroscopy and Imaging IV, 790503 (SPIE, San Francisco, CA, United States, 2011).

103. Guerrieri, F., Tisa, S. \& Zappa, F. Fast single-photon imager acquires 1024 pixels at $100 \mathrm{kframe} / \mathrm{s}$. In Proc. SPIE, Sensors, Cameras, and Systems for Industrial/Scientific Applications X, 72490 U (SPIE, San Jose, CA, United States, 2009).

104. Guerrieri, F., Tisa, S., Tosi, A. \& Zappa, F. Two-dimensional SPAD imaging camera for photon counting. IEEE Photonics J. 2, 759-774 (2010).

105. Kloster-Landsberg, M. et al. Multi-confocal fluorescence correlation spectroscopy in living cells using a complementary metal oxide semiconductorsingle photon avalanche diode array. Rev. Sci. Instrum. 84, 076105 (2013).

106. Singh, A. P. et al. The performance of $2 \mathrm{D}$ array detectors for light sheet based fluorescence correlation spectroscopy. Opt. Express 21, 8652-8668 (2013).

107. Carrara, L., Niclass, C., Scheidegger, N., Shea, H. \& Charbon, E. A gamma, X-ray and high energy proton radiation-tolerant CIS for space applications. In IEEE ISSCC Digest in Technical Paper 40-41 (IEEE, San Francisco, CA, United States, 2009).

108. Krieger, J. W. Mapping Diffusion Properties in Living Cells. Ph.D. thesis, Heidelberg Universität, Heidelberg, 2014.

109. Krieger, J. W. et al. Imaging fluorescence correlation: Novel results on new image sensors (SPAD arrays) and a comprehensive new software package (QUICKFIT 3.0). In Proc. Focus on Microscopy (FOM, Göttingen, Germany, 2015).

110. Buchholz, J. et al. Widefield high frame rate single-photon SPAD imagers for SPIM-FCS. Biophys. J. 114, 2455-2464 (2018).

111. Buchholz, J. Evaluation of Single Photon Avalanche Diode Arrays for Imaging Fluorescence Correlation Spectroscopy: FPGA-Based Data Readout and Fast Correlation Analysis on CPUs, GPUs and FPGAs. Ph.D. thesis, Heidelberg Universität, Heidelberg, 2016.

112. Michalet, $\mathrm{X}$. et al. Silicon photon-counting avalanche diodes for singlemolecule fluorescence spectroscopy. IEEE J. Sel. Top. Quantum Electron. 20, 248-267 (2014).

113. Rech, I., Resnati, D., Marangoni, S., Ghioni, M. \& Cova, S. Compact eightchannel photon counting module with monolithic array detector. In Proc.
SPIE, Advanced Photon Counting Techniques II, 677113 (SPIE, Boston, MA, United States, 2007).

114. Rech, I., Marangoni, S., Resnati, D., Ghioni, M. \& Cova, S. Multipixel singlephoton avalanche diode array for parallel photon counting applications. J. Mod. Opt. 56, 326-333 (2009).

115. Michalet, $X$. et al. High-throughput single-molecule fluorescence spectroscopy using parallel detection. In Proc. SPIE, Quantum Sensing and Nanophotonic Devices VII, 76082D (SPIE, San Francisco, CA, United States, 2010).

116. Michalet, X. et al. Development of new photon-counting detectors for singlemolecule fluorescence microscopy. Philos. Trans. R. Soc. Lond. Ser. B $\mathbf{3 6 8}$ 20120035 (2013).

117. Gulinatti, A., Ceccarelli, F., Rech, I. \& Ghioni, M. Silicon technologies for arrays of single photon avalanche diodes. In Proc. SPIE, Advanced Photon Counting Techniques X, 98580A (SPIE, Baltimore, MD, United States, 2016).

118. Ingargiola, A. et al. Parallel multispot smFRET analysis using an 8 pixel SPAD array. In Proc. SPIE, Single Molecule Spectroscopy and Superresolution Imaging V, 82280B (SPIE, San Francisco, CA, United States, 2012).

119. Ingargiola, A. et al. 8-spot smFRET analysis using two 8 pixel SPAD arrays. In Proc. SPIE, Single Molecule Spectroscopy and Superresolution Imaging VI, $85900 \mathrm{E}$ (SPIE, San Francisco, CA, United States, 2013).

120. Ingargiola, A. et al. Multispot single-molecule FRET: high-throughput analysis of freely diffusing molecules. PLOS ONE 12, e0175766 (2017).

121. Ingargiola, A. et al. 48-spot single-molecule FRET setup with periodic acceptor excitation. J. Chem. Phys. 148, 123304 (2018).

122. Abbe, E. Beiträge zur Theorie des Mikroskops und der mikroskopischen Wahrnehmung. Arch. für Mikrosk. Anat. 9, 413-418 (1873).

123. Hell, S. W. Microscopy and its focal switch. Nat. Methods 6, 24-32 (2009).

124. Fölling, J. et al. Fluorescence nanoscopy by ground-state depletion and single-molecule return. Nat. Methods 5, 943 (2008).

125. Antolovic, I. M., Burri, S., Bruschini, C., Hoebe, R. \& Charbon, E. Analyzing blinking effects in super resolution localization microscopy with singlephoton SPAD imagers. In Proc. SPIE, Single Molecule Spectroscopy and Superresolution Imaging IX, 971406 (SPIE, San Francisco, CA, United States, 2016).

126. Nieuwenhuizen, R. P. J. et al. Measuring image resolution in optical nanoscopy. Nat. Methods 10, 557-562 (2013).

127. Thompson, R. E., Larson, D. R. \& Webb, W. W. Precise nanometer localization analysis for individual fluorescent probes. Biophys. J. 82, 2775-2783 (2002).

128. Nahidiazar, L., Agronskaia, A. V., Broertjes, J., van den Broek, B. \& Jalink, K. Optimizing imaging conditions for demanding multi-color super resolution localization microscopy. PLoS ONE 11, e0158884 (2016).

129. Antolovic, I. M., Burri, S., Bruschini, C., Hoebe, R. A. \& Charbon, E. SPAD imagers for super resolution localization microscopy enable analysis of fast fluorophore blinking. Sci. Rep. 7, 44108 (2017).

130. Gyongy, I. et al. Smart-aggregation imaging for single molecule localization with SPAD cameras. Sci. Rep. 6, 1-10 (2016).

131. Krishnaswami, V., Van Noorden, C. J., Manders, E. M. \& Hoebe, R. A. Towards digital photon counting cameras for single-molecule optical nanoscopy. Opt. Nanosc. 3, 1 (2014).

132. Huang, F. et al. Video-rate nanoscopy using sCMOS camera-specific singlemolecule localization algorithms. Nat. Methods 10, 653 (2013).

133. Colthup, N. B., Daly, L. H. \& Wiberly, S. E. Introduction to Infrared and Raman Spectroscopy 2nd edn (Academic Press, Boston, MA, United States, 1975).

134. Krafft, C., Dietzek, B., Schmitt, M. \& Popp, J. Raman and coherent anti-Stokes Raman scattering microspectroscopy for biomedical applications. J. Biomed. Opt. 17, 040801 (2012).

135. Shipp, D., Sinjab, F. \& Notingher, I. Raman spectroscopy: techniques and applications in the life sciences. Adv. Opt. Photonics 9, 315-428 (2017).

136. Matousek, P. \& Stone, N. Development of deep subsurface Raman spectroscopy for medical diagnosis and disease monitoring. Chem. Soc. Rev. 45 1794-1802 (2016).

137. Nissinen, I. et al. A sub-ns time-gated CMOS single photon avalanche diode detector for Raman spectroscopy. In Proc. ESSDERC 375-378 (IEEE, Helsinki, Finland, 2011)

138. Kostamovaara, J. et al. Fluorescence suppression in Raman spectroscopy using a time-gated CMOS SPAD. Opt. Express 21, 31632-31645 (2013).

139. Maruyama, Y., Blacksberg, J. \& Charbon, E. A $1024 \times 8,700$ ps timegated SPAD line sensor for planetary surface exploration with laser Raman spectroscopy and LIBS. IEEE J. Solid-State Circuits 49, 179-189 (2014). 
140. Blacksberg, J., Maruyama, Y., Charbon, E. \& Rossman, G. R. Fast single-photon avalanche diode arrays for laser Raman spectroscopy. Opt. Lett. 36, 3672-3674 (2011).

141. Maruyama, Y., Blacksberg, J. \& Charbon, E. A $1024 \times 8700$ ps time-gated SPAD line sensor for laser Raman spectroscopy and LIBS in space and roverbased planetary exploration. In IEEE ISSCC Digest in Technical Paper 110-111 (IEEE, San Francisco, CA, United States, 2013).

142. Nissinen, I., Lansman, A.-K., Nissinen, J., Holma, J. \& Kostamovaara, J. $2 \times(4 \times) 128$ time-gated CMOS single photon avalanche diode line detector with 100 ps resolution for Raman spectroscopy. In Proc. ESSCIRC 291-294 (IEEE, Bucharest, Romania, 2013).

143. Nissinen, I. et al. A $2 \times(4) \times 128$ multitime-gated SPAD line detector for pulsed Raman spectroscopy. IEEE Sens. J. 15, 1358-1365 (2015).

144. Rojalin, T. et al. Fluorescence-suppressed time-resolved Raman spectroscopy of pharmaceuticals using complementary metal-oxide semiconductor (CMOS) single-photon avalanche diode (SPAD) detector. Anal. Bioanal. Chem. 408, 761-774 (2016)

145. Nissinen, I., Nissinen, J., Holma, J. \& Kostamovaara, J. A $4 \times 128$ SPAD array with a 78 ps 512 channel TDC for time-gated pulsed Raman spectroscopy. Analog Integr. Circuits Signal Process. 84, 353-362 (2015).

146. Holma, J., Nissinen, I., Nissinen, J. \& Kostamovaara, J. Characterization of the timing homogeneity in a CMOS SPAD array designed for time-gated Raman spectroscopy. IEEE Trans. Instrum. Meas. 66, 1837-1844 (2017).

147. Nissinen, I., Nissinen, J. \& Kostamovaara, J. Effects of the inhomogeneity of the time resolving CMOS single-photon avalanche diode array on time-gated Raman spectroscopy. In Proc. I2MTC 1-6 (IEEE, Turin, Italy, 2017).

148. Nissinen, I., Nissinen, J., Keränen, P., Stoppa, D. \& Kostamovaara, J. A $16 \times 256$ SPAD line detector with a 50-ps, 3-bit, 256-channel time-to-digital converter for Raman spectroscopy. IEEE Sens. J. 18, 3789-3798 (2018).

149. Alayed, M. \& Deen, M. Time-resolved diffuse optical spectroscopy and imaging using solid-state detectors: characteristics, present status, and research challenges. Sensors 17, 2115 (2017).

150. Ferocino, E. et al. High throughput detection chain for time domain optical mammography. Biomed. Opt. Express 9, 755-770 (2018).

151. Pavia, J. M., Wolf, M. \& Charbon, E. Single-photon avalanche diode imagers applied to near-infrared imaging. IEEE J. Sel. Top. Quantum Electron. 20, 291-298 (2014).

152. Pavia, J. M. Near-Infrared Optical Tomography with Single-Photon Avalanche Diode Image Sensors. Ph.D. thesis, EPFL, Lausanne, 2015.

153. Lindner, S. et al. Column-parallel dynamic TDC reallocation in SPAD sensor module fabricated in $180 \mathrm{~nm}$ CMOS for near infrared optical tomography. In Proc. IISW 86-89 (IISW, Hiroshima, Japan, 2017).

154. Kalyanov, A. et al. Time domain near-infrared optical tomography with timeof-flight SPAD camera: The new generation. In Proc. Biophotonics Congress: Biomedical Optics Congress OF4D-OF45 (Optical Society of America, Hollywood, FL, United States, 2018).

155. Mora, A. D. et al. Towards next-generation time-domain diffuse optics for extreme depth penetration and sensitivity. Biomed. Opt. Express 6, 1749-1760 (2015).

156. Stuker, F. et al. Hybrid small animal imaging system combining magnetic resonance imaging with fluorescence tomography using single photon avalanche diode detectors. IEEE Trans. Med. Imaging 30, 1265-1273 (2011)

157. Stuker, F. et al. A novel hybrid imaging system for simultaneous fluorescence molecular tomography and magnetic resonance imaging. In Proc. Biomedical Optics and 3-D Imaging BTuD1 (Optical Society of America, Miami, FL, United States, 2010).

158. Tanner, M. et al. Ballistic and snake photon imaging for locating optical endomicroscopy fibres. Biomed. Opt. Express 8, 4077-4095 (2017).

159. Muntean, A., Venialgo, E., Gnecchi, S., Jackson, C. \& Charbon, E. Towards a fully digital state-of-the-art analog SiPM. In Proc. NSS/MIC (IEEE, Atlanta, GA, United States, 2017).
160. Venialgo, E., Lusardi, N., Garzetti, F., Geraci, A. \& Charbon, E. A networkenabled PET detector module based on TDCs on FPGA. In Proc. NSS/MIC (IEEE, Atlanta, GA, United States, 2017).

161. Boiko, D. et al. A quantum imager for intensity correlated photons. New J. Phys. 11, 013001 (2009).

162. Boiko, D. et al. On the application of a monolithic array for detecting intensity-correlated photons emitted by different source types. Opt. Express 17, 15087-15103 (2009).

163. Unternährer, M., Bessire, B., Gasparini, L., Stoppa, D. \& Stefanov, A. Coincidence detection of spatially correlated photon pairs with a monolithic timeresolving detector array. Opt. Express 24, 28829-28841 (2016).

164. Gasparini, L. et al. SUPERTWIN: towards 100 kpixel CMOS quantum image sensors for quantum optics applications. In Proc. SPIE, Quantum Sensing and Nano Electronics and Photonics XIV, $101112 \mathrm{~L}$ (SPIE, San Francisco, CA, United States, 2017).

165. Aull, B. Geiger-mode avalanche photodiode arrays integrated to all-digital CMOS circuits. Sensors 16, 495 (2016).

166. Aull, B. F. et al. A study of crosstalk in a $256 \times 256$ photon counting imager based on silicon Geiger-mode avalanche photodiodes. IEEE Sens. J. 15, 2123-2132 (2015).

167. Pavia, J. M., Scandini, M., Lindner, S., Wolf, M. \& Charbon, E. A $1 \times 400$ backside-illuminated SPAD sensor with $49.7 \mathrm{ps}$ resolution, $30 \mathrm{pJ} / \mathrm{sample}$ TDCs fabricated in 3D CMOS technology for near-infrared optical tomography. IEEE J. Solid-State Circuits 50, 2406-2418 (2015).

168. Durini, D. et al. BackSPAD - back-side illuminated single-photon avalanche diodes: concept and preliminary performances. In Proc. NSS/MIC 1-2 (IEEE, Anaheim, CA, United States, 2012).

169. Zou, Y., Bronzi, D., Villa, F. \& Weyers, S. Backside Illuminated Wafer-to-Wafer Bonding Single Photon Avalanche Diode Array. In Proc. PRIME 1-4 (IEEE, Grenoble, France, 2014).

170. Nolet, F. et al. A 2D proof of principle towards a 3D digital SiPM in HV CMOS with low output capacitance. IEEE Trans. Nucl. Sci. 63, 2293-2299 (2016).

171. Nolet, F. et al. Digital SiPM channel integrated in CMOS 65 nm with 17.5 ps FWHM single photon timing resolution. Nucl. Instrum. Methods Phys. Res. Sect. A 912, 29-32 (2018).

172. Bérubé, B.-L. et al. Implementation study of single photon avalanche diodes (SPAD) in $0.8 \mu \mathrm{m}$ HV CMOS technology. IEEE Trans. Nucl. Sci. 62, 710-718 (2015).

173. Al Abbas, T. et al. Backside illuminated SPAD image sensor with $7.83 \mu \mathrm{m}$ pitch in 3D-Stacked CMOS technology. In Proc. IEDM 8.1.1-8.1.4 (IEEE, San Francisco, CA, United States, 2016).

174. Lindner, S. et al. A high-PDE, backside-illuminated SPAD in 65/40 nm 3D IC CMOS pixel with cascoded passive quenching and active recharge. IEEE Electron Device Lett. 38, 1547-1550 (2017).

175. Lee, M.-J. et al. A back-illuminated 3D-stacked single-photon avalanche diode in 45 nm CMOS technology. In Proc. IEDM 16.6.1-16.6.4 (IEEE, San Francisco, CA, United States, 2017).

176. You, Z., Parmesan, L., Pellegrini, S. \& Henderson, R. K. $3 \mu \mathrm{m}$ pitch, $1 \mu \mathrm{m}$ active diameter SPAD arrays in $130 \mathrm{~nm}$ CMOS imaging technology. In Proc. IISW 238-241 (IISW, Hiroshima, Japan, 2017).

177. Pellegrini, S. \& Rae, B. Fully industrialised single photon avalanche diodes. In Proc. SPIE, Advanced Photon Counting Techniques XI, 102120D (SPIE, Anaheim, CA, United States, 2017).

178. Antolovic, I. M. et al. Photon-counting arrays for time-resolved imaging Sensors 16, 1005 (2016)

179. Ximenes, A. R. et al. A $256 \times 25645 / 65 \mathrm{~nm}$ 3D-stacked SPAD-based direct TOF image sensor for LiDAR applications with optical polar modulation for up to $18.6 \mathrm{~dB}$ interference suppression. In IEEE ISSCC Digest in Technical Paper 96-98 (IEEE, San Francisco, CA, United States, 2018).

180. Krieger, J. W. et al. Imaging fluorescence (cross-)correlation spectroscopy in live cells and organisms. Nat. Protoc. 10, 1948-1974 (2015). 